\title{
TITLE:
}

\section{A rarefied gas flow caused by a discontinuous wall temperature}

$\operatorname{AUTHOR}(S):$

Aoki, K; Takata, S; Aikawa, H; Golse, F

CITATION:

Aoki, K ... [et al]. A rarefied gas flow caused by a discontinuous wall temperature. PHYSICS OF FLUIDS 2001, 13(9): 2645-2661

ISSUE DATE:

2001-09

URL:

http://hdl.handle.net/2433/39780

\section{RIGHT:}

Copyright 2001 American Institute of Physics. This article may be downloaded for personal use only. Any other use requires prior permission of the author and the American Institute of Physics. 


\title{
A rarefied gas flow caused by a discontinuous wall temperature
}

\author{
Kazuo Aoki, Shigeru Takata, and Hidefumi Aikawa \\ Department of Aeronautics and Astronautics, Graduate School of Engineering, Kyoto University, \\ Kyoto 606-8501, Japan \\ François Golse \\ Département de Mathématiques et Applications, Ecole Normale Supérieure, 75230 Paris Cedex 05, France \\ and U. F. R. de Mathématiques, Université Paris VII, 75251 Paris Cedex 05, France
}

(Received 21 February 2000; accepted 27 April 2001)

\begin{abstract}
A flow of a rarefied gas caused by a discontinuous wall temperature is investigated on the basis of kinetic theory in the following situation. The gas is confined in a two-dimensional square container, and the left and right halves of the wall of the container are kept at different uniform temperatures, so that the temperatures of the top and bottom walls are discontinuous at their respective middle points. External forces are assumed to be absent. The steady flow of the gas induced in the container by the effect of the discontinuities is analyzed numerically on the basis of the Bhatnagar-GrossKrook model of the Boltzmann equation and the diffuse reflection boundary condition by means of an accurate finite-difference method. The features of the flow are clarified for a wide range of the Knudsen number. In particular, it is shown that, as the Knudsen number becomes small (i.e., as the system approaches the continuum limit), the maximum flow speed tends to approach a finite value, but the region with appreciable flow shrinks to the points of discontinuity; thus, the overall flow in the container vanishes nonuniformly in the continuum limit. The behavior of the molecular velocity distribution function is also investigated in detail. (c) 2001 American Institute of Physics.
\end{abstract}

[DOI: $10.1063 / 1.1389283$ ]

\section{INTRODUCTION}

Let us consider an ideal gas around solid boundaries at rest with arbitrary but steady temperature distributions. We assume that there is no external force in the field and also that the gas is at rest and its pressure is uniform at infinity when an infinite domain is considered. If we investigate the steady behavior of the gas on the basis of the (compressible) Navier-Stokes system (i.e., the conservation equations of mass, momentum, and energy with Newton's law of stress and Fourier's law of heat flow and the boundary condition of nonslip or nonjump type), we find that $\mathbf{v}=0$ and $p=$ const, where $\mathbf{v}$ is the flow velocity and $p$ is the pressure, are the obvious solution of the continuity and momentum equations satisfying the nonslip boundary condition for the velocity. Then the temperature field is determined by the energy equation, which reduces to the steady heat-conduction equation for the temperature, and the nonjump boundary condition. This fact indicates that for any temperature distribution of the boundaries, no flow is induced in the gas. The NavierStokes system is generally accepted as the correct system to describe the behavior of a gas in the continuum limit where the Knudsen number vanishes. Here, the Knudsen number is the ratio of the mean free path of the gas molecules to the characteristic length of the system. Therefore, it is concluded that no steady flow is induced by the temperature field in the continuum limit. This conclusion, drawn from the NavierStokes system, is correct in spite of the fact that the NavierStokes system has a serious defect in describing the behavior of a gas even in the continuum limit. We will come back to this point at the end of this section.
In contrast, in a rarefied gas where the Knudsen number is not vanishingly small, the situation is different. The temperature field can cause a steady flow of the gas without the help of external forces. Such flows have extensively been investigated on the basis of kinetic theory for a wide range of the Knudsen number. ${ }^{1-22}$ In particular, in the case of small Knudsen numbers (i.e., the case near the continuum limit), the features of the flow have been understood systematically. To be more specific, for small Knudsen numbers, a general theory (asymptotic theory) ${ }^{23-30}$ that describes the steady behavior of the gas around arbitrarily shaped boundaries by means of a system of fluid-dynamic-type equations and slip boundary conditions (and the Knudsen layer correction near the boundary) has been established by a systematic asymptotic analysis of the Boltzmann equation and its kinetic boundary condition. According to the theory, the flow induced by the temperature field is classified into the following three types: (i) thermal creep flow, ${ }^{1-7}$ (ii) thermal stress slip flow, ${ }^{24,8-11}$ and (iii) nonlinear thermal stress flow. ${ }^{12,13}$ The flow (i) is induced along the boundary from the colder part to the hotter when the temperature of the boundary is not uniform. The flow (ii) is induced along the boundary when the temperature gradient normal to the boundary in the gas is not uniform along the boundary. The flow (iii) is induced in the gas when the space between isothermal surfaces varies along the surfaces. The flow speed, divided by a quantity of the order of the sound speed, is of the order of the Knudsen number for the flows (i) and (iii) and the Knudsen number squared for the flow (ii). In the systems where the deviation from an equilibrium state at rest is small, the flow (iii) is 
negligibly small compared to the flows (i) and (ii).

The asymptotic theory mentioned above is based on the assumption that the local Knudsen number, the local mean free path divided by the local length scale of variation of physical quantities, is uniformly small. This means that the radius of curvature of the boundary should be much larger than the mean free path, and the boundary condition specified on the boundary should be smooth enough. If this condition is not fulfilled, therefore, there is a possibility that flows other than the above three types are caused by the temperature field even when the Knudsen number is small.

Such an example is given in Refs. 31 and 32. Let us now consider a flat plate placed in a rarefied gas in a container kept at a uniform temperature and suppose that the plate is heated or cooled uniformly. Then, although the thermal creep flow [the flow (i)] is absent because of the uniform temperature of the plate and of the container, a rather strong and localized flow is induced around the edges of the plate. This is due to the following fact. Although the temperature of the plate itself is uniform, there arises a steep temperature gradient in the gas along the plate near the edges because of the presence of the edges. The temperature gradient then causes a flow by the same mechanism as the thermal creep flow (see Refs. 28-30). This new type of flow was first found by the numerical computations ${ }^{31,32}$ by the use of the direct simulation Monte Carlo (DSMC) method $^{33,34}$ and then verified experimentally. ${ }^{32}$ A rough estimate in Ref. 32 shows that the local flow speed near the edges, divided by a quantity of the order of the sound speed, is likely to be of the order of $\mathrm{Kn}^{1 / 2}$ for small $\mathrm{Kn}$, where $\mathrm{Kn}$ is the overall Knudsen number. This fact was confirmed ${ }^{35}$ with a reasonable accuracy in a subsequent finite-difference analysis based on the BhatnagarGross-Krook (BGK) model ${ }^{36-38}$ of the Boltzmann equation. Therefore, the flow has a stronger effect than the flows (i)(iii).

A similar localized and steep temperature gradient along the boundary arises when the temperature of the boundary changes abruptly along it, as in the case of a discontinuous temperature distribution. Also in this case, a flow that cannot be covered by the asymptotic theory is expected to be induced along the boundary even when the overall Knudsen number is small and the boundary is geometrically smooth. In the present study, we are going to investigate such a flow, i.e., a flow induced by a discontinuous wall temperature. More specifically, we consider a gas in a two-dimensional square container, the wall of which has a discontinuous and sectionally uniform temperature distribution (see Sec. II A for the details). We investigate the steady behavior of the gas, especially the flow induced around the point of discontinuity, numerically for a wide range of the Knudsen number with special interest in the behavior for small Knudsen numbers. Making use of the BGK model of the Boltzmann equation and the diffuse reflection condition as our basic system, we carry out an accurate numerical analysis by means of a finite-difference method that is able to describe the behavior of the discontinuity in the molecular velocity distribution function introduced by the discontinuity in the boundary temperature (see Sec. III).

We conclude this section with a brief discussion on the

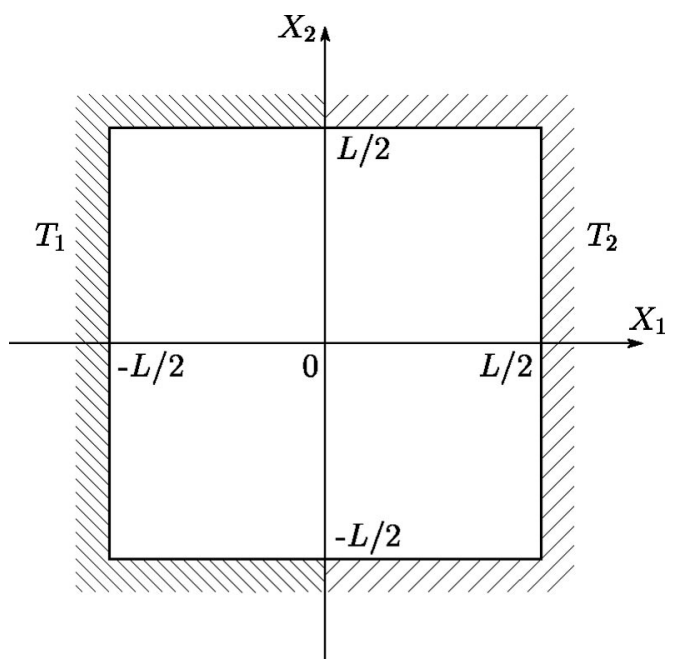

FIG. 1. A rarefied gas in a two-dimensional square container with a discontinuous wall temperature.

validity of the Navier-Stokes system in the continuum limit. A recent study ${ }^{26}$ based on kinetic theory showed that, in the situation considered in the first paragraph of this section, though $\mathbf{v}=0$ and $p=$ const are correct, the steady heatconduction equation does not give the correct temperature field even in the continuum limit. This is due to the fact that gas flows of the order of the Knudsen number, which therefore vanish in the continuum limit, give a finite effect on the temperature distribution in this limit. Since it is an effect of the flows that do not exist in the continuum fluid dynamics, it was termed the ghost effect. ${ }^{39,22}$ This effect is particularly important because it reveals the fatal defect contained in the Navier-Stokes system for a gas. The effect manifests itself in a wide class of problems. The reader is referred to Refs. 29, 30, and 40-44 in addition to Refs. 26, 39, and 22 for further information.

\section{FORMULATION OF THE PROBLEM}

\section{A. Problem}

Let us consider a rarefied gas confined in a twodimensional square container $-L / 2 \leqslant X_{1} \leqslant L / 2,-L / 2 \leqslant X_{2}$ $\leqslant L / 2$, where $X_{i}$ is a rectangular coordinate system (Fig. 1). The left half $\left(X_{1}<0\right)$ and the right half $\left(X_{1}>0\right)$ of the wall of the container are kept at different uniform temperatures $T_{1}$ and $T_{2}$, respectively. Therefore, the temperatures of the top and bottom walls are discontinuous at their respective middle points $\left(X_{1}=0, X_{2}= \pm L / 2\right)$. External forces are assumed to be absent. We investigate the steady flow of the gas induced in the container by the effect of the discontinuities of the wall temperature, for a wide range of the Knudsen number, on the basis of kinetic theory. Our basic assumptions are as follows: (i) the behavior of the gas is described by the BGK model $^{36-38}$ of the Boltzmann equation; (ii) the gas molecules are reflected diffusely on the wall of the container. 


\section{B. Basic equation}

The BGK model of the Boltzmann equation in the present steady and spatially two-dimensional problem is written as ${ }^{28,45}$

$$
\begin{aligned}
& \xi_{1} \frac{\partial f}{\partial X_{1}}+\xi_{2} \frac{\partial f}{\partial X_{2}}=A_{c} \rho\left(f_{e}-f\right), \\
& f_{e}=\frac{\rho}{(2 \pi R T)^{3 / 2}} \exp \left(-\frac{\left(\xi_{i}-v_{i}\right)^{2}}{2 R T}\right), \\
& \rho=\int f d \xi \\
& v_{i}=\frac{1}{\rho} \int \xi_{i} f d \xi \\
& T=\frac{1}{3 R \rho} \int\left(\xi_{i}-v_{i}\right)^{2} f d \boldsymbol{\xi},
\end{aligned}
$$

where $\xi_{i}$ is the molecular velocity, $d \boldsymbol{\xi}=d \xi_{1} d \xi_{2} d \xi_{3}$, $f\left(X_{1}, X_{2}, \xi_{i}\right)$ is the velocity distribution function of the gas molecules, $\rho\left(X_{1}, X_{2}\right)$ is the density of the gas, $v_{i}$ $=\left(v_{1}\left(X_{1}, X_{2}\right), v_{2}\left(X_{1}, X_{2}\right), 0\right)$ is its flow velocity, $T\left(X_{1}, X_{2}\right)$ is its temperature, $R$ is the gas constant per unit mass, and $A_{c}$ is a constant $\left(A_{c} \rho\right.$ is the collision frequency of a gas molecule). The domain of integration with respect to $\xi_{i}$ in Eqs. (3a) $-(3 c)$ and in Eqs. (9b) and (9c) below is its whole space.

The boundary condition on the wall of the container is written as follows: ${ }^{28,45}$

$$
\begin{aligned}
& f=\frac{\rho_{w}}{\left(2 \pi R T_{w}\right)^{3 / 2}} \exp \left(-\frac{\xi_{i}^{2}}{2 R T_{w}}\right) \quad\left(\xi_{j} n_{j}>0\right), \\
& \rho_{w}=-\left(\frac{2 \pi}{R T_{w}}\right)^{1 / 2} \int_{\xi_{j} n_{j}<0} \xi_{j} n_{j} f d \boldsymbol{\xi},
\end{aligned}
$$

where $n_{i}$ is the unit vector normal to the wall pointing into the gas, and

$$
T_{w}=\left\{\begin{array}{cc}
T_{1} & \text { for } \quad\left(-\frac{L}{2} \leqslant X_{1}<0, \quad X_{2}= \pm \frac{L}{2}\right) \\
& \text { or } \quad\left(X_{1}=-\frac{L}{2}, \quad-\frac{L}{2}<X_{2}<\frac{L}{2}\right), \\
T_{2} & \text { for } \quad\left(0<X_{1} \leqslant \frac{L}{2}, \quad X_{2}= \pm \frac{L}{2}\right) \\
& \text { or } \quad\left(X_{1}=\frac{L}{2}, \quad-\frac{L}{2}<X_{2}<\frac{L}{2}\right) .
\end{array}\right.
$$

The problem is symmetric with respect to the $X_{1}$ axis. Therefore, we can analyze the problem only in the lower half $\left(-L / 2 \leqslant X_{2} \leqslant 0\right)$ of the container by imposing the specular reflection condition on the $X_{1}$ axis, namely,

$$
\begin{aligned}
f\left(X_{1}, 0, \xi_{1}, \xi_{2}, \xi_{3}\right)= & f\left(X_{1}, 0, \xi_{1},-\xi_{2}, \xi_{3}\right) \\
& \text { for } \xi_{2}<0, \quad\left(-\frac{L}{2}<X_{1}<\frac{L}{2}\right) .
\end{aligned}
$$

Then, the solution in the upper half $\left(0<X_{2} \leqslant L / 2\right)$ is given by

$$
\begin{aligned}
f\left(X_{1}, X_{2}, \xi_{1}, \xi_{2}, \xi_{3}\right)= & f\left(X_{1},-X_{2}, \xi_{1},-\xi_{2}, \xi_{3}\right) \\
& \text { for }\left(-\frac{L}{2} \leqslant X_{1} \leqslant \frac{L}{2}, \quad 0<X_{2} \leqslant \frac{L}{2}\right),
\end{aligned}
$$

in terms of that in the lower half.

The pressure $p\left(X_{1}, X_{2}\right)$, stress tensor $p_{i j}\left(X_{1}, X_{2}\right)\left(p_{13}\right.$ $\left.=p_{23}=0\right)$, and heat-flow vector $q_{i}\left(X_{1}, X_{2}\right)\left(q_{3}=0\right)$ are expressed as

$$
\begin{aligned}
& p=R \rho T, \\
& p_{i j}=\int\left(\xi_{i}-v_{i}\right)\left(\xi_{j}-v_{j}\right) f d \boldsymbol{\xi}, \\
& q_{i}=\frac{1}{2} \int\left(\xi_{i}-v_{i}\right)\left(\xi_{j}-v_{j}\right)^{2} f d \boldsymbol{\xi} .
\end{aligned}
$$

\section{Dimensionless variables}

Let us now introduce the following dimensionless variables:

$$
\begin{aligned}
& x_{i}=\frac{X_{i}}{L}, \quad \zeta_{i}=\frac{\xi_{i}}{\left(2 R T_{1}\right)^{1 / 2}}, \\
& \hat{f}=\frac{\left(2 R T_{1}\right)^{3 / 2}}{\rho_{\mathrm{av}}} f, \quad \hat{\rho}=\frac{\rho}{\rho_{\mathrm{av}}}, \\
& \hat{v}_{i}=\frac{v_{i}}{\left(2 R T_{1}\right)^{1 / 2}}, \quad \hat{T}=\frac{T}{T_{1}}, \quad \hat{p}=\frac{p}{R \rho_{\mathrm{av}} T_{1}}, \\
& \hat{p}_{i j}=\frac{p_{i j}}{R \rho_{\mathrm{av}} T_{1}}, \quad \hat{q}_{i}=\frac{q_{i}}{\left(\rho_{\mathrm{av}} / 2\right)\left(2 R T_{1}\right)^{3 / 2}},
\end{aligned}
$$

where $\rho_{\mathrm{av}}$ is the average density of the gas in the container. Then, the BGK equation, Eqs. (1)-(3c), is written in the following dimensionless form:

$$
\begin{aligned}
& \zeta_{1} \frac{\partial \hat{f}}{\partial x_{1}}+\zeta_{2} \frac{\partial \hat{f}}{\partial x_{2}}=\frac{2}{\pi^{1 / 2} \mathrm{Kn}} \hat{\rho}\left(\hat{f}_{e}-\hat{f}\right), \\
& \hat{f}_{e}=\frac{\hat{\rho}}{(\pi \hat{T})^{3 / 2}} \exp \left(-\frac{\left(\zeta_{i}-\hat{v}_{i}\right)^{2}}{\hat{T}}\right), \\
& \hat{\rho}=\int \hat{f} d \zeta, \\
& \hat{v}_{i}=\frac{1}{\hat{\rho}} \int \zeta_{i} \hat{f} d \zeta, \\
& \hat{T}=\frac{2}{3 \hat{\rho}} \int\left(\zeta_{i}-\hat{v}_{i}\right)^{2} \hat{f} d \zeta, \\
& \mathrm{Kn}=2\left(2 R T_{1} / \pi\right)^{1 / 2}\left(A_{c} \rho_{\mathrm{av}} L\right)^{-1}=l_{1} / L, \\
& d \zeta=d \zeta_{1} d \zeta_{2} d \zeta_{3},
\end{aligned}
$$

where $\mathrm{Kn}$ is the Knudsen number, and $l_{1}$ is the mean free path of the gas molecules in the equilibrium state at rest with temperature $T_{1}$ and density $\rho_{\mathrm{av}}$. The domain of integration 
with respect to $\zeta_{i}$ in Eqs. (13a)-(13c) and Eqs. (19b) and (19c) below is its whole space. On the other hand, the dimensionless form of the boundary condition (4)-(6) restricted in the lower half of the container is

$$
\begin{aligned}
& \hat{f}=\frac{\hat{\rho}_{w}}{\left(\pi \hat{T}_{w}\right)^{3 / 2}} \exp \left(-\frac{\zeta_{i}^{2}}{\hat{T}_{w}}\right) \quad\left(\zeta_{j} n_{j}>0\right), \\
& \hat{\rho}_{w}=-\frac{2 \pi^{1 / 2}}{\hat{T}_{w}^{1 / 2}} \int_{\zeta_{j} n_{j}<0} \zeta_{j} n_{j} \hat{f} d \zeta,
\end{aligned}
$$

with

$$
\hat{T}_{w}=\left\{\begin{array}{lll}
1 & \text { for } \quad\left(-\frac{1}{2} \leqslant x_{1}<0, x_{2}=-\frac{1}{2}\right) \\
& \text { or } \quad\left(x_{1}=-\frac{1}{2},-\frac{1}{2}<x_{2}<0\right), \\
T_{2} / T_{1} & \text { for } \quad\left(0<x_{1} \leqslant \frac{1}{2}, x_{2}=-\frac{1}{2}\right) \\
& \text { or } \quad\left(x_{1}=\frac{1}{2},-\frac{1}{2}<x_{2}<0\right),
\end{array}\right.
$$

and that of the condition (7) is

$$
\begin{aligned}
\hat{f}\left(x_{1}, 0, \zeta_{1}, \zeta_{2}, \zeta_{3}\right)= & \hat{f}\left(x_{1}, 0, \zeta_{1},-\zeta_{2}, \zeta_{3}\right) \\
& \text { for } \zeta_{2}<0,\left(-\frac{1}{2}<x_{1}<\frac{1}{2}\right) .
\end{aligned}
$$

The dimensionless forms of Eqs. (9a)-(9c) are given by

$$
\begin{aligned}
& \hat{p}=\hat{\rho} \hat{T}, \\
& \hat{p}_{i j}=2 \int\left(\zeta_{i}-\hat{v}_{i}\right)\left(\zeta_{j}-\hat{v}_{j}\right) \hat{f} d \zeta, \\
& \hat{q}_{i}=\int\left(\zeta_{i}-\hat{v}_{i}\right)\left(\zeta_{j}-\hat{v}_{j}\right)^{2} \hat{f} d \zeta .
\end{aligned}
$$

\section{Further transformation}

By means of a standard method, ${ }^{46}$ we can eliminate the $x_{3}$ component, $\zeta_{3}$, of the molecular velocity from the system, Eqs. (11)-(18). That is, if we multiply Eqs. (11), (15), and (18) by 1 and $\zeta_{3}^{2}$ and integrate the respective results over the whole range of $\zeta_{3}$, we obtain two simultaneous integrodifferential equations and their boundary conditions. To summarize the result, we first introduce the following (nondimensional) marginal velocity distribution functions $\hat{g}\left(x_{1}, x_{2}, \zeta_{1}, \zeta_{2}\right)$ and $\hat{h}\left(x_{1}, x_{2}, \zeta_{1}, \zeta_{2}\right)$ and column vector $\boldsymbol{\Phi}\left(x_{1}, x_{2}, \zeta_{1}, \zeta_{2}\right)$ composed of $\hat{g}$ and $\hat{h}$ :

$$
\boldsymbol{\Phi}=\left[\begin{array}{l}
\hat{g} \\
\hat{h}
\end{array}\right]=\int_{-\infty}^{\infty}\left[\begin{array}{c}
1 \\
\zeta_{3}^{2}
\end{array}\right] \hat{f} d \zeta_{3} .
$$

The simultaneous equations for $\boldsymbol{\Phi}$, derived from Eq. (11), are given by

$$
\begin{aligned}
& \zeta_{1} \frac{\partial \boldsymbol{\Phi}}{\partial x_{1}}+\zeta_{2} \frac{\partial \boldsymbol{\Phi}}{\partial x_{2}}=\frac{2}{\pi^{1 / 2} \mathrm{Kn}} \hat{\rho}\left(\boldsymbol{\Phi}_{e}-\boldsymbol{\Phi}\right), \\
& \boldsymbol{\Phi}_{e}=\frac{\hat{\rho}}{2 \pi} \exp \left(-\frac{\left(\zeta_{1}-\hat{v}_{1}\right)^{2}+\left(\zeta_{2}-\hat{v}_{2}\right)^{2}}{\hat{T}}\right)\left[\begin{array}{c}
2 \hat{T}^{-1} \\
1
\end{array}\right],
\end{aligned}
$$

$$
\begin{aligned}
\hat{\rho}= & \int_{-\infty}^{\infty} \int_{-\infty}^{\infty} \hat{g} d \zeta_{1} d \zeta_{2}, \\
\hat{v}_{i}= & \frac{1}{\hat{\rho}} \int_{-\infty}^{\infty} \int_{-\infty}^{\infty} \zeta_{i} \hat{g} d \zeta_{1} d \zeta_{2} \quad(i=1,2), \\
\hat{T}= & \frac{2}{3 \hat{\rho}} \int_{-\infty}^{\infty} \int_{-\infty}^{\infty}\left\{\left[\left(\zeta_{1}-\hat{v}_{1}\right)^{2}+\left(\zeta_{2}-\hat{v}_{2}\right)^{2}\right] \hat{g}+\hat{h}\right\} \\
& \times d \zeta_{1} d \zeta_{2} .
\end{aligned}
$$

The boundary condition on the wall of the container, derived from Eq. (15), is

$$
\begin{aligned}
& \boldsymbol{\Phi}=\frac{\hat{\rho}_{w}}{2 \pi} \exp \left(-\frac{\zeta_{1}^{2}+\zeta_{2}^{2}}{\hat{T}_{w}}\right)\left[\begin{array}{c}
2 \hat{T}_{w}^{-1} \\
1
\end{array}\right] \quad\left(\zeta_{1} n_{1}+\zeta_{2} n_{2}>0\right), \\
& \hat{\rho}_{w}=-\frac{2 \pi^{1 / 2}}{\hat{T}_{w}} \int_{\zeta_{1} n_{1}+\zeta_{2} n_{2}<0}\left(\zeta_{1} n_{1}+\zeta_{2} n_{2}\right) \hat{g} d \zeta_{1} d \zeta_{2},
\end{aligned}
$$

where $\hat{T}_{w}$ is defined in Eq. (17), and the symmetry condition on the $x_{1}$ axis, derived from Eq. (18), is

$$
\begin{aligned}
\boldsymbol{\Phi}\left(x_{1}, 0, \zeta_{1}, \zeta_{2}\right)= & \boldsymbol{\Phi}\left(x_{1}, 0, \zeta_{1},-\zeta_{2}\right) \\
& \text { for } \zeta_{2}<0, \quad\left(-\frac{1}{2}<x_{1}<\frac{1}{2}\right) .
\end{aligned}
$$

The $\hat{p}_{i j}\left(\hat{p}_{13}=\hat{p}_{23}=0\right)$ and $\hat{q}_{i}\left(\hat{q}_{3}=0\right)$ in Eqs. (19b) and (19c) are written as

$$
\begin{aligned}
\hat{p}_{i j}=2 \int_{-\infty}^{\infty} \int_{-\infty}^{\infty}\left(\zeta_{i}-\hat{v}_{i}\right)\left(\zeta_{j}-\hat{v}_{j}\right) \hat{g} d \zeta_{1} d \zeta_{2} \quad(i, j=1,2) \\
\hat{p}_{33}=2 \int_{-\infty}^{\infty} \int_{-\infty}^{\infty} \hat{h} d \zeta_{1} d \zeta_{2} \\
\hat{q}_{i}=\int_{-\infty}^{\infty} \int_{-\infty}^{\infty}\left(\zeta_{i}-\hat{v}_{i}\right)\left\{\left[\left(\zeta_{1}-\hat{v}_{1}\right)^{2}+\left(\zeta_{2}-\hat{v}_{2}\right)^{2}\right] \hat{g}+\hat{h}\right\} \\
\quad \times d \zeta_{1} d \zeta_{2} \quad(i=1,2)
\end{aligned}
$$

\section{NUMERICAL ANALYSIS}

We analyze Eqs. (21)-(26) numerically by a finitedifference method. One of the difficulties in the numerical analysis arises from the fact that the velocity distribution function is discontinuous in the gas. In this section, we first discuss it briefly and then give an outline of the numerical method.

\section{A. Discontinuity in velocity distribution function}

If the velocity distribution function at a point in space, say $X_{i}^{(0)}$, is discontinuous at a certain molecular velocity, say $\xi_{i}^{(0)}$, then the discontinuity propagates in the direction of $\xi_{i}^{(0)}$ from $X_{i}^{(0)}$ (i.e., along the characteristic of the Boltzmann equation). ${ }^{47}$ Such a propagation of discontinuity is commonly observed in the gas around a convex boundary, namely, the velocity distribution function is generally discon- 
tinuous there. In the present problem, the discontinuous boundary condition causes the discontinuity in the velocity distribution function in the gas, as seen below. For a general discussion on the discontinuity, including its relation to the Knudsen layer and Sone layer ${ }^{48}$ for small Knudsen numbers, the reader is referred to Ref. 47 (a brief discussion is also found in Ref. 39). The propagation of discontinuity is also discussed in the framework of propagation phenomena associated with the Boltzmann equation in a recent paper by Cercignani. ${ }^{49}$

Let us consider the velocity distribution function of the gas molecules leaving the bottom wall $\left(X_{2}=-L / 2\right.$ or $x_{2}$ $=-1 / 2)$ in the present problem. At the point of discontinuity of the wall temperature $\left(x_{1}=0, x_{2}=-1 / 2\right)$, the limit from the left $\left[\boldsymbol{\Phi}\left(0_{-},-1 / 2, \zeta_{1}, \zeta_{2}\right)\right]$ is prescribed by the boundary condition (24) and (25) $\left(n_{1}=0, n_{2}=1\right)$ with $\hat{T}_{w}=1$, whereas the limit from the right $\left[\boldsymbol{\Phi}\left(0_{+},-1 / 2, \zeta_{1}, \zeta_{2}\right)\right]$ is prescribed by Eqs. (24) and (25) $\left(n_{1}=0, n_{2}=1\right)$ with $\hat{T}_{w}=T_{2} / T_{1}$. (Here we are considering the dimensionless marginal velocity distribution functions $\boldsymbol{\Phi}$, but the situation is essentially the same for the original velocity distribution function $\hat{f}$ or f.) Therefore, these two limits, in general, do not coincide $\left[\boldsymbol{\Phi}\left(0_{-},-1 / 2, \zeta_{1}, \zeta_{2}\right) \neq \boldsymbol{\Phi}\left(0_{+},-1 / 2, \zeta_{1}, \zeta_{2}\right)\right]$ for any fixed molecular velocity $\left(\zeta_{1}, \zeta_{2}\right)\left(\zeta_{2}>0\right)$. This discontinuity propagates in the gas in the direction of $\left(\zeta_{1}, \zeta_{2}\right)$, i.e., along the characteristic of Eq. (21). Therefore, at a point $\left(x_{1}, x_{2}\right)$ in the gas, the velocity distribution function is generally discontinuous in the direction $\zeta_{1} / \zeta_{2}=x_{1} /\left(x_{2}+1 / 2\right)$ in the $\zeta_{1} \zeta_{2}$ plane. It is easily shown that the discontinuity attenuates over the distance of the order of the molecular free path in its propagation because of the effect of molecular collisions. ${ }^{47}$ These properties are essentially the same as those of the discontinuity originating from the leading and trailing edges in the case of a rarefied gas flow past a (thickless) flat plate. ${ }^{50}$ In the present problem, the velocity distribution function at $\left(x_{1}, x_{2}\right)$ is also discontinuous in the direction $\zeta_{1} / \zeta_{2}$ $=x_{1} /\left(x_{2}-1 / 2\right)$ because of the presence of the discontinuity in the temperature of the top wall at $x_{1}=0, x_{2}=1 / 2$. In the framework of the boundary-value problem, Eqs. (21), (24), and (26), in the lower half of the container, the second discontinuity corresponds to the discontinuity propagating in the direction $\zeta_{1} / \zeta_{2}=x_{1} /\left(-x_{2}+1 / 2\right)$ from the middle point of the bottom wall $\left(x_{1}=0, x_{2}=-1 / 2\right)$, reflected on the specularly reflecting boundary $\left(x_{2}=0\right)$, and reaching the point $\left(x_{1}, x_{2}\right)\left(x_{2}<0\right)$. The discontinuity point of the boundary temperature is also a singular point for the macroscopic variables in the sense that their limiting values at the point are different depending on the direction of approach (see Ref. 50).

It should be mentioned that the discontinuity of the velocity distribution function is also caused by the four corners of the container. At these points, the limit of $\hat{\rho}_{w}$ [Eq. (25)] from the bottom or the top wall is generally different from that from the side wall because the domain of integration as well as the integrand in Eq. (25) is different. Therefore, the boundary condition (24) is discontinuous there. These discontinuities also propagate into the gas. However, the discontinuities caused by the corners are much smaller than those caused by the discontinuities of the wall temperature. (Note that the discontinuities in $\hat{\rho}_{w}$ at the corners disappear when the velocity distribution of incident molecules there is isotropic and that the velocity distribution approaches a local Maxwellian near each corner for small Knudsen numbers.)

Finally, we make a brief comment on mathematical theory for the propagation of discontinuities. The above discussion about the behavior of the discontinuities is based on the assumption that the gain term of the collision integral of the Boltzmann equation is continuous inside the gas region (in the case of the BGK model, this is equivalent to assuming that the density, flow velocity, and temperature are continuous there). Although it is plausible, to show this continuity rigorously is a difficult mathematical problem even for the BGK model that is much simpler than the original Boltzmann equation. In time-dependent problems, discontinuities (or, more generally, singularities) contained in the velocity distribution function at the initial time also propagate in the gas, attenuating because of molecular collisions, as time goes on. Reference 51 deals with such propagation successfully with mathematical rigor on the basis of the Boltzmann equation. However, it shows that the propagating singularities can be discriminated only from a slightly less singular remainder. In Ref. 52, on the other hand, the discontinuities induced by discontinuous boundary data are studied mathematically for a simple one-speed linear transport equation which has a similar structure to the linearized BGK model, and it is proved that the gain term is continuous and the discontinuities can be separated from a continuous remainder. The mathematical results obtained for this simple transport equation are consistent with the situation described in the second paragraph in this subsection. ${ }^{53}$

\section{B. Outline of numerical analysis}

The finite-difference methods that are capable of describing the correct behavior of the discontinuity of the velocity distribution function have been devised and developed in Refs. 54-58, and 50 in various situations. The type of propagation of the discontinuity in the present problem is almost the same as that in Ref. 50, where a supersonic rarefied gas flow past a flat plate is investigated. Therefore, we can exploit the finite-difference scheme developed there with a slight modification. Since the detailed description of the method is found in Ref. 50, we give only a brief outline of the method.

(i) For the numerical analysis, we restrict the $\zeta_{1} \zeta_{2}$ plane to a finite domain $\left|\zeta_{1}\right| \leqslant Z_{1},\left|\zeta_{2}\right| \leqslant Z_{2}$, where $Z_{1}$ and $Z_{2}$ are positive constants chosen in such a way that $\boldsymbol{\Phi}$ is negligibly small at $\left|\zeta_{1}\right| \simeq Z_{1}$ and $\left|\zeta_{2}\right| \simeq Z_{2}$. The discrete solution $\boldsymbol{\Phi}_{\#}$ of $\boldsymbol{\Phi}$ at the lattice points in the $\left(x_{1}, x_{2}, \zeta_{1}, \zeta_{2}\right)$ space is constructed as the limit of the sequence $\boldsymbol{\Phi}_{\#}^{(n)}$, where $\boldsymbol{\Phi}_{\#}^{(n)}$ denotes the $\boldsymbol{\Phi}_{\#}$ at the $n$th step of iteration; the $\boldsymbol{\Phi}_{\#}^{(n)}$ is obtained as follows by the use of a finite-difference equation, corresponding to Eq. (21), that gives a relation between $\boldsymbol{\Phi}_{\#}^{(n)}$ and $\boldsymbol{\Phi}_{\#}^{(n-1)}$. Let $\left(\zeta_{1}^{(k)}, \zeta_{2}^{(l)}\right)$ denote the lattice points in the $\zeta_{1} \zeta_{2}$ plane. We choose appropriate initial distributions $\boldsymbol{\Phi}_{\#}^{(0)}$. Let $\boldsymbol{\Phi}_{\#}^{(n-1)}$ be known. For each $\zeta_{1}^{(k)}>0, \boldsymbol{\Phi}_{\#}^{(n)}$ is determined from $x_{1}=-1 / 2$ to $1 / 2$ and from $x_{2}=-1 / 2$ to 0 (or from $x_{2}=0$ to 


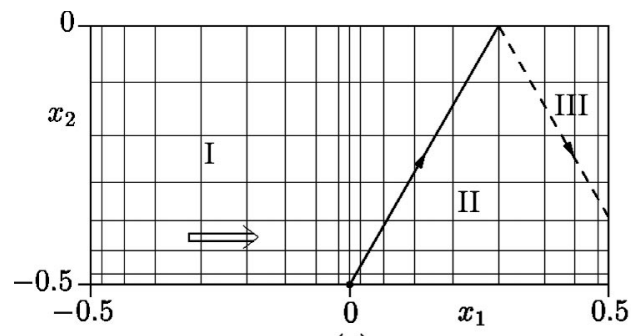

(a)

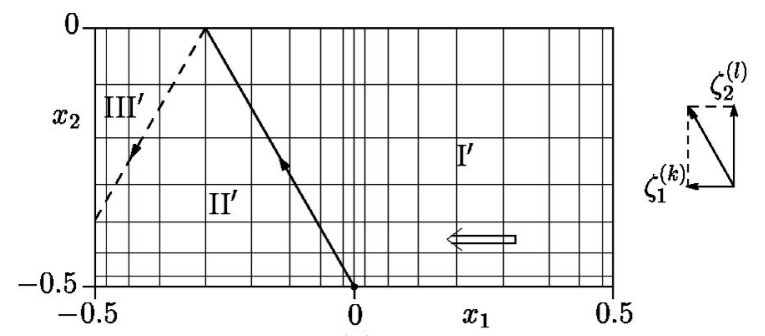

(b)

FIG. 2. Schematic figure for the procedure of numerical computation. (a) $\zeta_{1} \geqslant 0$, (b) $\zeta_{1}<0$.
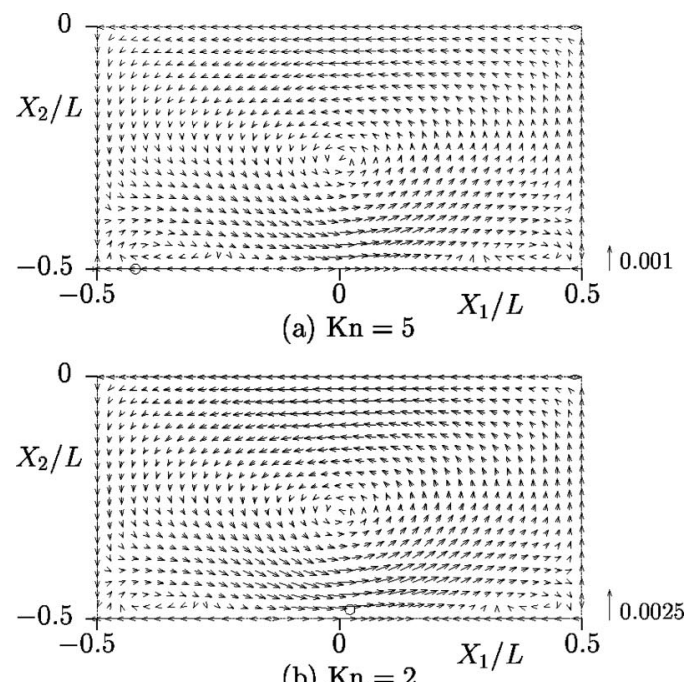

(b) $K n=2$

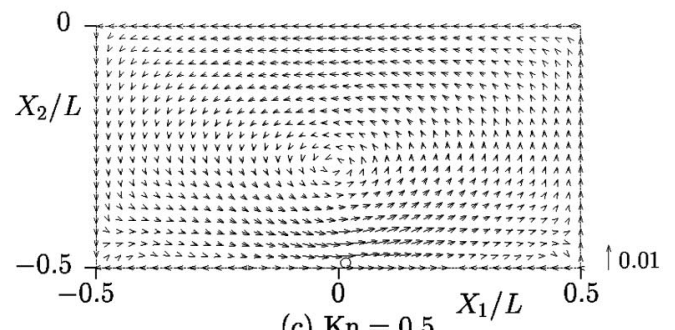

(c) $\mathrm{Kn}=0.5$

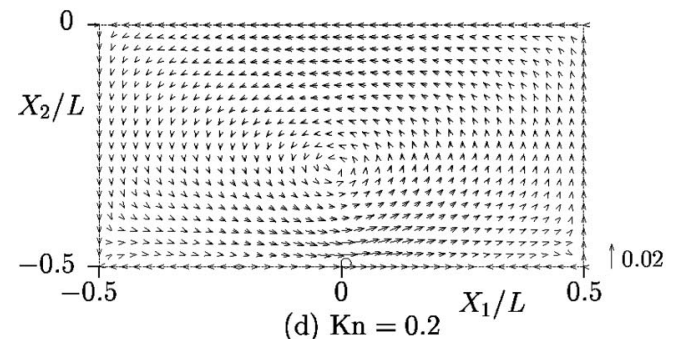

FIG. 3. Flow induced in the lower half of the container for $T_{2} / T_{1}=2$. (a) $\mathrm{Kn}=5$, (b) $\mathrm{Kn}=2$, (c) $\mathrm{Kn}=0.5$, (d) $\mathrm{Kn}=0.2$. The arrow indicates the twodimensional flow velocity vector $\left(v_{1}, v_{2}\right) /\left(2 R T_{1}\right)^{1 / 2}$ at its starting point. The reference length of the arrow is shown in the right margin of each figure. The symbol $\bigcirc$ indicates the point with the maximum flow speed.
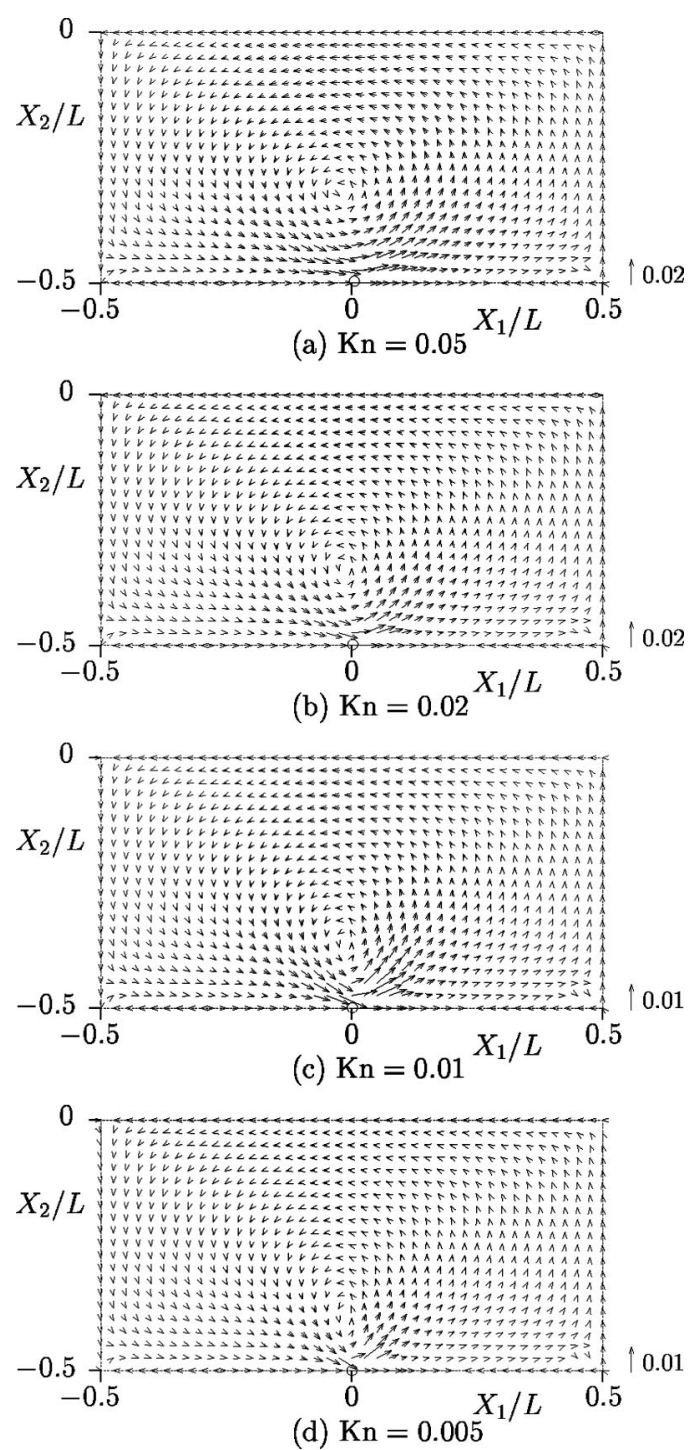

FIG. 4. Flow induced in the lower half of the container for $T_{2} / T_{1}=2$. (a) $\mathrm{Kn}=0.05$, (b) $\mathrm{Kn}=0.02$, (c) $\mathrm{Kn}=0.01$, (d) $\mathrm{Kn}=0.005$. See the caption of Fig. 3.

$-1 / 2$ ) by the use of the finite-difference equation, the boundary condition on the left wall, and the boundary condition on the bottom wall (or the specular reflection condition on $x_{2}=0$ ) for all $\zeta_{2}^{(l)} \geqslant 0$ (or for all $\zeta_{2}^{(l)}<0$ ) [Fig. 2(a)]. Similarly, for each $\zeta_{1}^{(k)}<0, \boldsymbol{\Phi}_{\#}^{(n)}$ is determined from $x_{1}$ $=1 / 2$ to $-1 / 2$ and from $x_{2}=-1 / 2$ to 0 (or from $x_{2}=0$ to $-1 / 2$ ) by the use of the finite-difference equation, the boundary condition on the right wall, and the boundary condition on the bottom wall (or the specular reflection condition on $x_{2}=0$ ) for all $\zeta_{2}^{(l)} \geqslant 0$ (or for all $\zeta_{2}^{(l)}<0$ ) [Fig. 2(b)]. For $\zeta_{1}^{(k)}=0, \boldsymbol{\Phi}_{\#}^{(n)}$ is determined from $x_{2}=-1 / 2$ to 0 (or from $x_{2}=0$ to $-1 / 2$ ) by the use of the finite-difference equation and the boundary condition on the bottom wall (or the specular reflection condition on $x_{2}=0$ ) for all $\zeta_{2}^{(l)} \geqslant 0$ (or for all $\left.\zeta_{2}^{(l)}<0\right)$.

(ii) As explained in the preceding subsection, $\boldsymbol{\Phi}$ is discontinuous in the gas. Here, we neglect the discontinuities caused by the four corners of the container (see the third paragraph of Sec. III A; the discontinuities of $\hat{\rho}_{w}$ at the cor- 


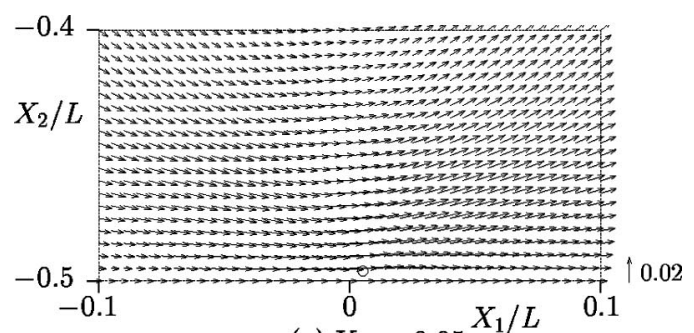

(a) $\mathrm{Kn}=0.05^{X_{1} / L}$

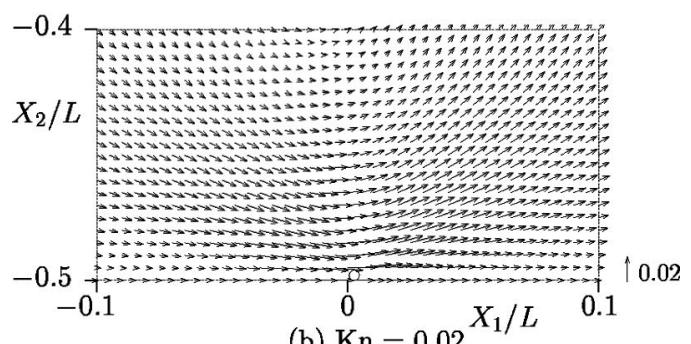

(b) $\mathrm{Kn}=0.02$

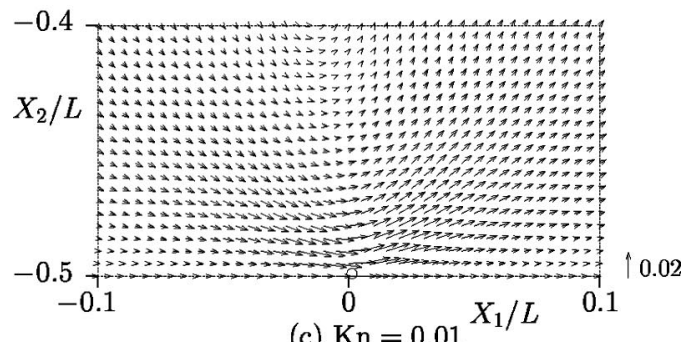

(c) $\mathrm{Kn}=0.01$

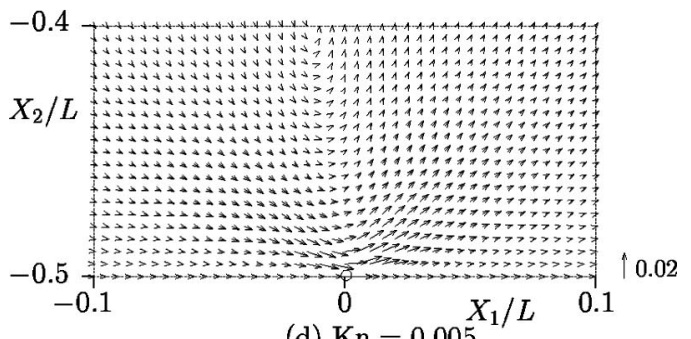

(d) $\mathrm{Kn}=0.005$

FIG. 5. Flow near the point of discontinuity for $T_{2} / T_{1}=2$. Magnified figure of the range $-0.1 \leqslant X_{1} / L \leqslant 0.1,-0.5 \leqslant X_{2} / L \leqslant-0.4$ of Fig 4 . (a) $\mathrm{Kn}$ $=0.05$, (b) $\mathrm{Kn}=0.02$, (c) $\mathrm{Kn}=0.01$, (d) $\mathrm{Kn}=0.005$. See the caption of Fig. 3.

ners can be estimated from the numerical result, and it turns out to be negligibly small for small $\mathrm{Kn}$ ). Then, for $\left(\zeta_{1}^{(k)}, \zeta_{2}^{(l)}\right)=(a, b) \quad\left[\right.$ or $\left.\quad\left(\zeta_{1}^{(k)}, \zeta_{2}^{(l)}\right)=(a,-b)\right] \quad(a>0, \quad b$ $>0), \boldsymbol{\Phi}$ is discontinuous along the line $x_{2}=(b / a) x_{1}-1 / 2$ [or $x_{2}=-(b / a) x_{1}+1 / 2$ ], i.e., the boundary between the regions I and II (or II and III) in Fig. 2(a). When we discretize Eq. (21), we should not apply finite-difference approximation to the derivative terms $\partial \boldsymbol{\Phi} / \partial x_{1}$ and $\partial \boldsymbol{\Phi} / \partial x_{2}$ across the discontinuity. Therefore, we need to make a local correction in the finite-difference scheme. That is, we first separate the regions I and II+ III (or I+ II and III) in Fig. 2(a) and then use the same finite-difference equation as in (i) in each region. Since these two regions are disconnected, we need, as the boundary condition for the region II+ III (or III), the limiting value of $\boldsymbol{\Phi}$ from the right along the line of discontinuity in the process of determining $\boldsymbol{\Phi}_{\#}^{(n)}$ from $x_{1}=-1 / 2$ to $1 / 2$ in the procedure (i). This limiting value is obtained separately with the aid of another finite-difference equation for

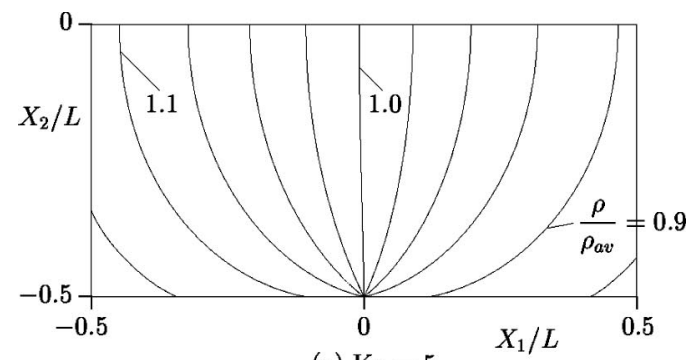

(a) $\mathrm{Kn}=5$

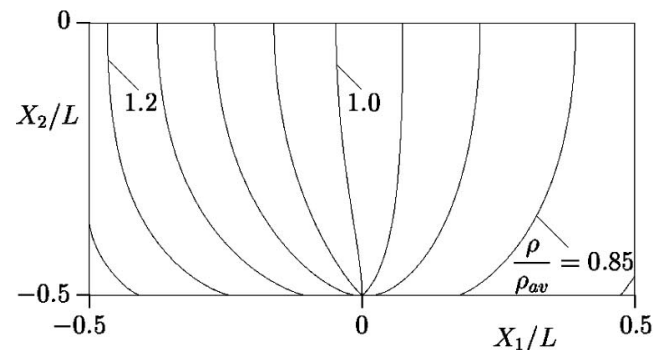

(b) $\mathrm{Kn}=0.5$

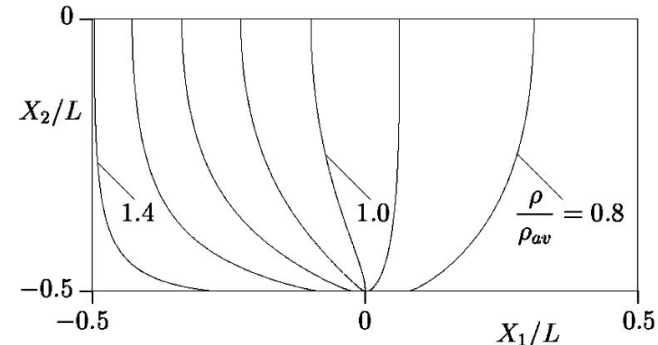

(c) $\mathrm{Kn}=0.05$

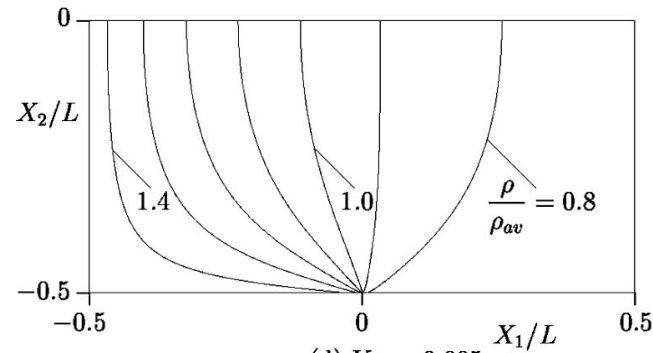

(d) $\mathrm{Kn}=0.005$

FIG. 6. Isolines of the density for $T_{2} / T_{1}=2$. (a) $\mathrm{Kn}=5 \quad\left(\rho / \rho_{\mathrm{av}}=0.875\right.$ $+0.025 m ; m=0,1, \ldots, 10)$, (b) $\mathrm{Kn}=0.5\left(\rho / \rho_{\mathrm{av}}=0.8+0.05 m ; m=0,1\right.$, $\ldots, 9),\left(\right.$ c) $\mathrm{Kn}=0.05\left(\rho / \rho_{\mathrm{av}}=0.8+0.1 m ; m=0,1, \ldots, 6\right)$, (d) $\mathrm{Kn}$ $=0.005\left(\rho / \rho_{\mathrm{av}}=0.8+0.1 m ; m=0,1, \ldots, 6\right)$.

Eq. (21) along (the right-hand side of) the discontinuity line. The treatment for $\left(\zeta_{1}^{(k)}, \zeta_{2}^{(l)}\right)=\left(a^{\prime}, b^{\prime}\right)$ [or $\left(\zeta_{1}^{(k)}, \zeta_{2}^{(l)}\right)$ $\left.=\left(a^{\prime},-b^{\prime}\right)\right]\left(a^{\prime}<0, b^{\prime}>0\right)$ is essentially the same. That is, we decompose the original domain into two regions $\mathrm{I}^{\prime}$ and $\mathrm{II}^{\prime}+\mathrm{III}^{\prime}$ (or $\mathrm{I}^{\prime}+\mathrm{II}^{\prime}$ and $\mathrm{III}^{\prime}$ ) in Fig. 2(b) by the discontinuity line $x_{2}=\left(b^{\prime} / a^{\prime}\right) x_{1}-1 / 2\left[\right.$ or $\left.x_{2}=-\left(b^{\prime} / a^{\prime}\right) x_{1}+1 / 2\right]$ and apply the same finite-difference equation as in (i) in each region. In this case, we need, as the boundary condition for the region $\mathrm{II}^{\prime}+\mathrm{III}^{\prime}$ (or $\mathrm{III}^{\prime}$ ), the limiting value of $\boldsymbol{\Phi}$ from the left along the line of discontinuity in the process of determining $\boldsymbol{\Phi}_{\#}^{(n)}$ from $x_{1}=1 / 2$ to $-1 / 2$ in the procedure (i). This limiting value is obtained separately with the aid of a finite-difference equation for Eq. (21) along (the left-hand side of) the discontinuity line. 


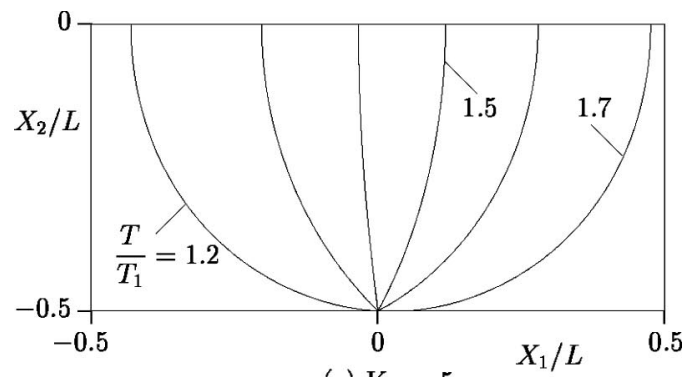

(a) $\mathrm{Kn}=5$

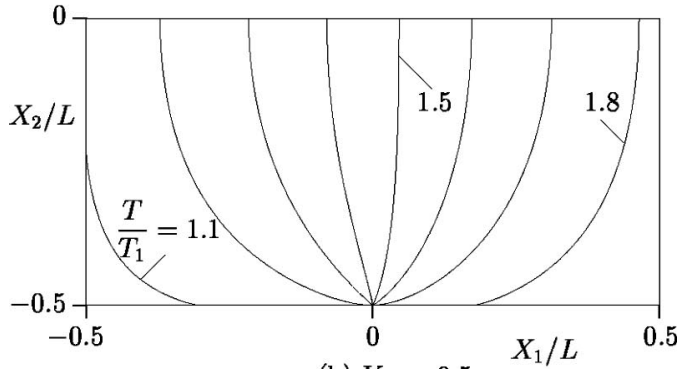

(b) $\mathrm{Kn}=0.5$

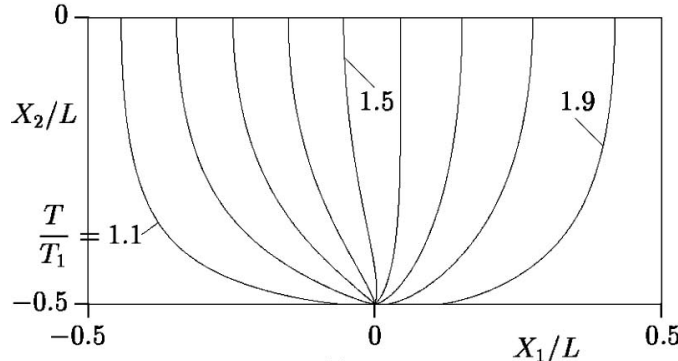

(c) $\mathrm{Kn}=0.05$

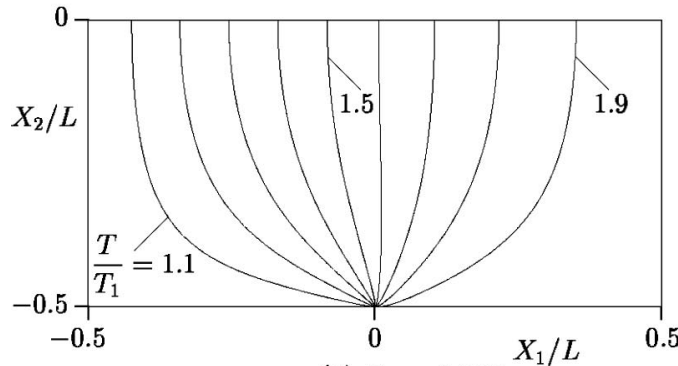

(d) $\mathrm{Kn}=0.005$

FIG. 7. Isolines of the temperature for $T_{2} / T_{1}=2$. (a) $\mathrm{Kn}=5\left(T / T_{1}=1.2\right.$ $+0.1 m ; m=0,1, \ldots, 5)$, (b) $\mathrm{Kn}=0.5\left(T / T_{1}=1.1+0.1 m ; m=0,1, \ldots\right.$, 7), (c) $\mathrm{Kn}=0.05\left(T / T_{1}=1.1+0.1 m ; m=0,1, \ldots, 8\right)$, (d) $\mathrm{Kn}=0.005$ $\left(T / T_{1}=1.1+0.1 m ; m=0,1, \ldots, 8\right)$.

\section{RESULTS OF NUMERICAL ANALYSIS}

The computation has been carried out for a fixed temperature ratio $T_{2} / T_{1}=2$ and for various values of the Knudsen number in the range $0.005 \leqslant \mathrm{Kn} \leqslant 5$. In this section, we show and discuss the results of the computation.

\section{A. Flow induced in the gas}

The flow velocity field in the lower half of the container in the case of $T_{2} / T_{1}=2$ is shown in Figs. 3 and 4 for various values of $\mathrm{Kn}$ in its descending order, i.e., for $\mathrm{Kn}=5,2,0.5$, and 0.2 in Figs. 3(a) $-3(\mathrm{~d})$ and for $\mathrm{Kn}=0.05,0.02,0.01$, and 0.005 in Figs. 4(a)-4(d). The arrow in the figures indicates the two-dimensional flow velocity vector $\left(v_{1}, v_{2}\right)$ normal-

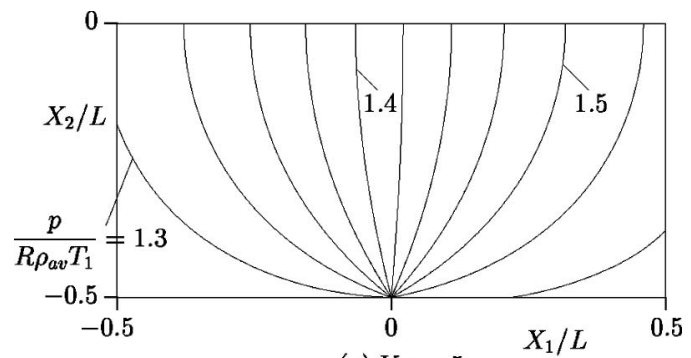

(a) $\mathrm{Kn}=5$

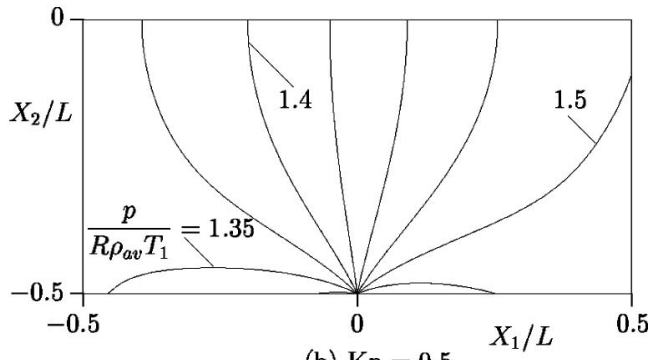

(b) $\mathrm{Kn}=0.5$

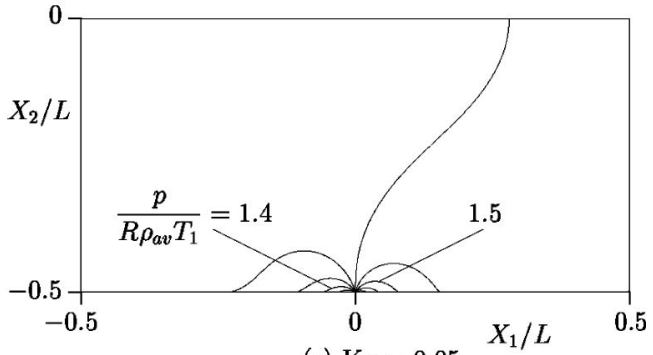

(c) $\mathrm{Kn}=0.05$

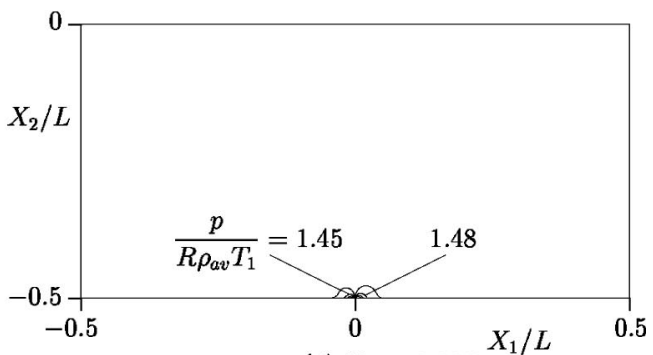

(d) $\mathrm{Kn}=0.005^{X_{1} / L}$

FIG. 8. Isolines of the pressure for $T_{2} / T_{1}=2$. (a) $\mathrm{Kn}=5\left(p / R \rho_{\mathrm{av}} T_{1}=1.3\right.$ $+0.025 m ; m=0,1, \ldots, 10)$, (b) $\mathrm{Kn}=0.5\left(p / R \rho_{\mathrm{av}} T_{1}=1.35+0.025 m ; m\right.$ $=0,1, \ldots, 6)$, (c) $\mathrm{Kn}=0.05\left(p / R \rho_{\mathrm{av}} T_{1}=1.38+0.02 m ; m=0,1, \ldots, 8\right)$, (d) $\mathrm{Kn}=0.005\left(p / R \rho_{\mathrm{av}} T_{1}=1.42+0.01 m ; m=0,1, \ldots, 9\right)$.

ized by $\left(2 R T_{1}\right)^{1 / 2}$, and its length corresponding to 0.001 , $0.0025,0.01$, or 0.02 is shown in the right margin of each figure (note the difference in the scale of the arrow); the point at which the flow speed takes the maximum is indicated by $\bigcirc$. Recall that the wall temperature is discontinuous at $X_{1} / L=0, X_{2} / L=-0.5$.

For all Kn in Figs. 3 and 4, a counterclockwise circulating flow is observed. The flow, which is very weak at $\mathrm{Kn}$ $=5$, is intensified as $\mathrm{Kn}$ decreases (Fig. 3). But, as Kn decreases to 0.05 and to 0.02 [Figs. 4(a) and 4(b)], though the flow near the point of discontinuity of the wall temperature remains of the same order of magnitude, the flow in the other part becomes weaker. With further decrease of Kn [Figs. 4(c) and 4(d)], the region where the flow is appreciable shrinks to the close neighborhood of the point of discontinuity, and the 


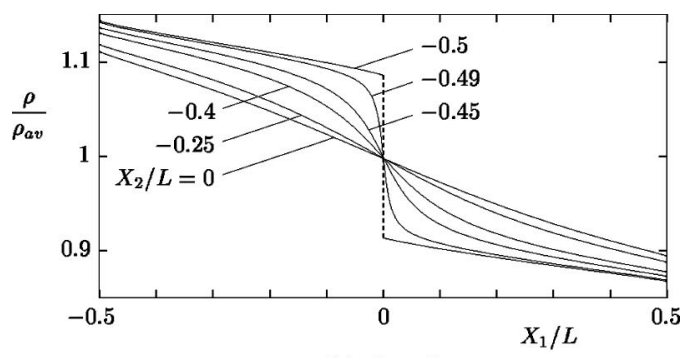

(a) $\mathrm{Kn}=5$

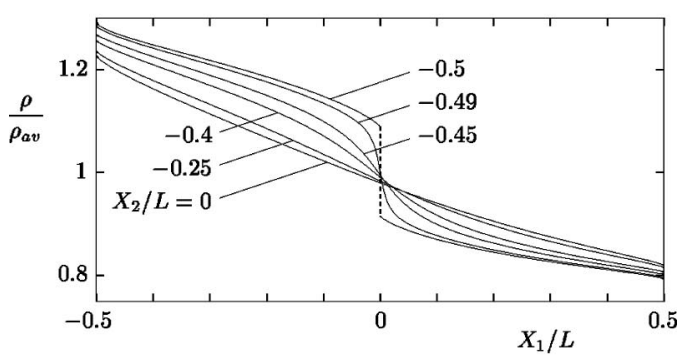

(b) $\mathrm{Kn}=0.5$

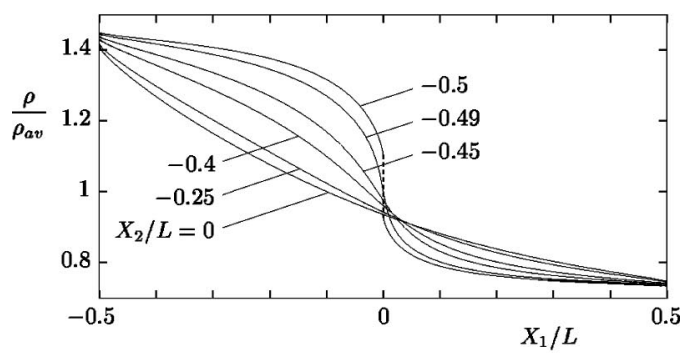

(c) $\mathrm{Kn}=0.05$

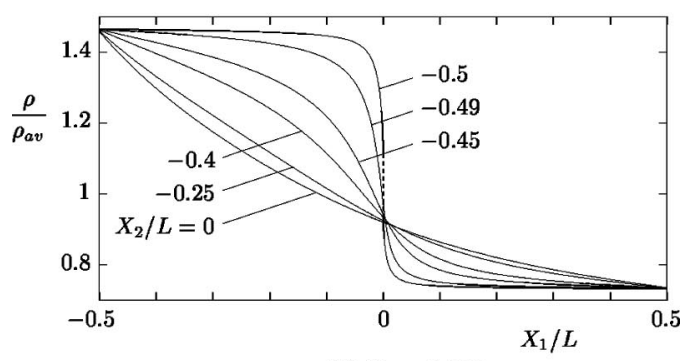

(d) $\mathrm{Kn}=0.005$

FIG. 9. Distributions of the density along the lines $X_{2} / L=$ const for $T_{2} / T_{1}=2$. (a) $\mathrm{Kn}=5$, (b) $\mathrm{Kn}=0.5$, (c) $\mathrm{Kn}=0.05$, (d) $\mathrm{Kn}=0.005$.

flow itself seems to be weakened. When $\mathrm{Kn}$ is relatively large ( $\mathrm{Kn}=5$ and 2), a flow toward the left is observed on the bottom wall in the parts near the side walls, and it induces two weak clockwise circulating flows at both bottom corners. Although the leftward flow is seen for all $\mathrm{Kn}$, it becomes weaker and thus the two circulating flows become weaker and smaller as $\mathrm{Kn}$ becomes small. The maximum speed is attained on the wall far from the point of discontinuity (i.e., in the leftward flow) for $\mathrm{Kn}=5$, whereas it is attained in the gas near the point of discontinuity for other $\mathrm{Kn}$. As $\mathrm{Kn}$ becomes small, the point with the maximum speed approaches the point of discontinuity.

In order to see the local structure of the flow field of Fig. 4 near the point of discontinuity, we show in Figs. 5(a)-5(d) the magnified figures of the part $-0.1 \leqslant X_{1} / L \leqslant 0.1,-0.5$ $\leqslant X_{2} / L \leqslant-0.4$ of Figs. 4(a)-4(d). For $\mathrm{Kn}=0.005$ [Fig.

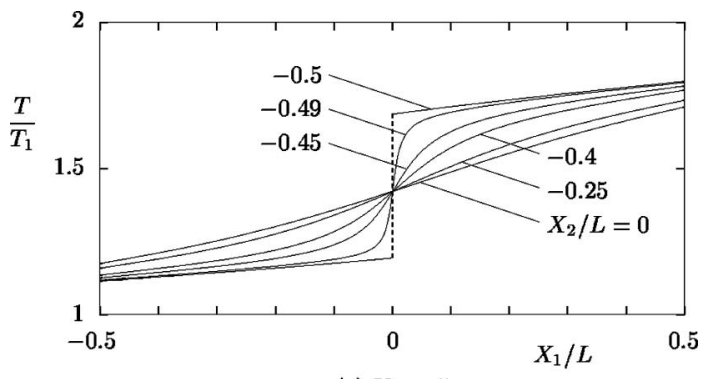

(a) $\mathrm{Kn}=5$

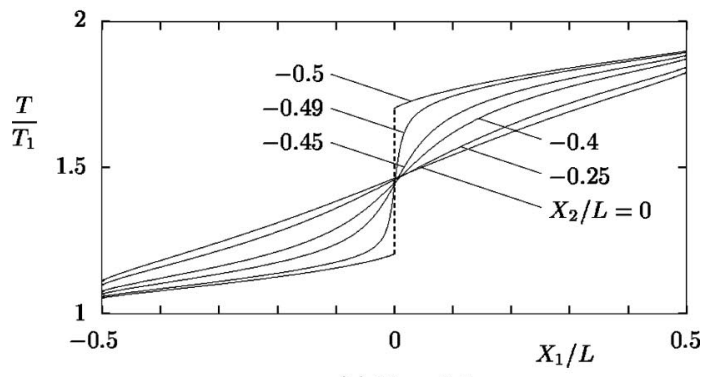

(b) $\mathrm{Kn}=0.5$

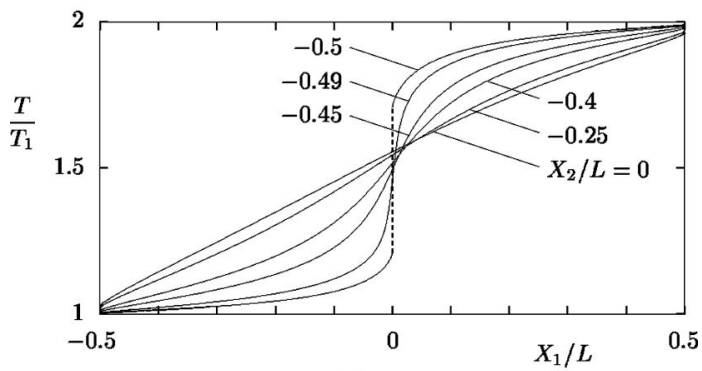

(c) $\mathrm{Kn}=0.05$

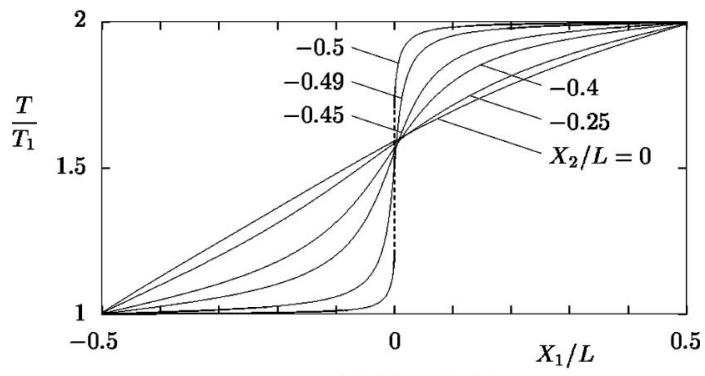

(d) $\mathrm{Kn}=0.005$

FIG. 10. Distributions of the temperature along the lines $X_{2} / L=$ const for $T_{2} / T_{1}=2$. (a) $\mathrm{Kn}=5$, (b) $\mathrm{Kn}=0.5$, (c) $\mathrm{Kn}=0.05$, (d) $\mathrm{Kn}=0.005$.

$5(\mathrm{~d})$ ], the flow is very localized even in this small region and almost vanishes on the outer edge of the region. However, the flow speed in the vicinity of the discontinuity point is not slow in comparison with that for other Kn [Figs. 5(a)-5(c)].

In the case of free-molecular gas $(\mathrm{Kn}=\infty)$, where the effect of collisions between gas molecules is neglected, no flow is induced in the gas. This fact was proved by Sone ${ }^{59,60}$ in a very general situation (arbitrary shape, arrangement, and temperature distribution of the boundary, the Maxwell-type diffuse-specular reflection condition with an accommodation coefficient varying along the boundary, etc.). On the other hand, as mentioned in Sec. I, no flow is induced by the temperature field in the continuum limit $\left(\mathrm{Kn}=0_{+}\right)$. How- 


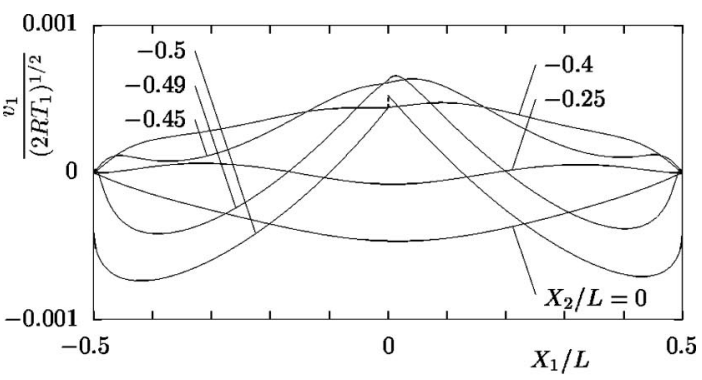

(a) $\mathrm{Kn}=5$

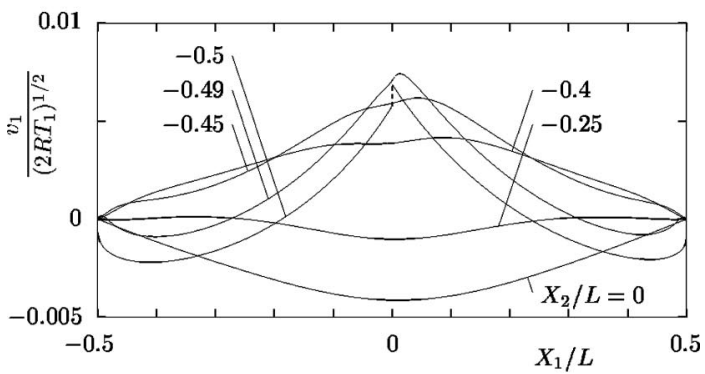

(b) $\mathrm{Kn}=0.5$

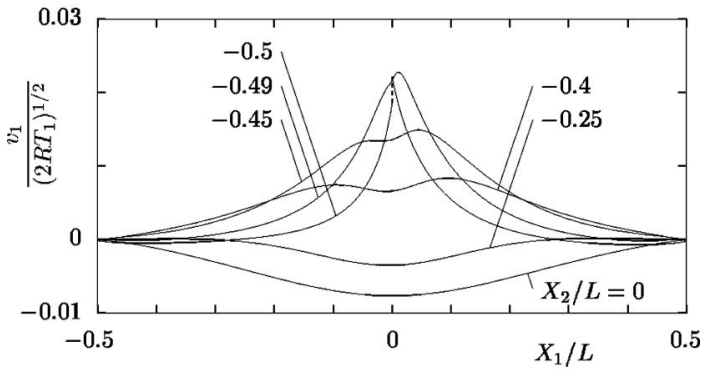

(c) $\mathrm{Kn}=0.05$

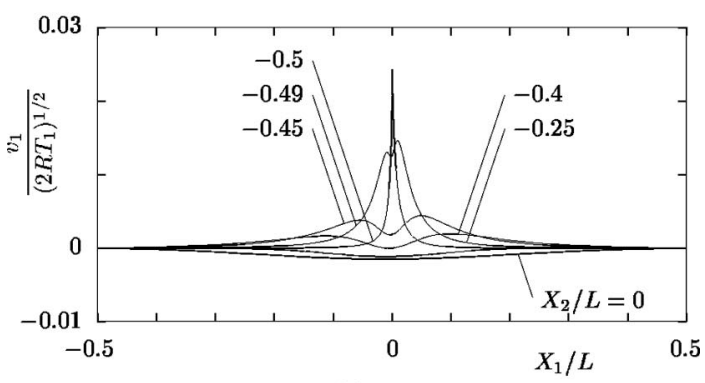

(d) $\mathrm{Kn}=0.005$

FIG. 11. Distributions of the $X_{1}$ component of the flow velocity along the lines $X_{2} / L=$ const for $T_{2} / T_{1}=2$. (a) $\mathrm{Kn}=5$, (b) $\mathrm{Kn}=0.5$, (c) $\mathrm{Kn}=0.05$, (d) $\mathrm{Kn}=0.005$.

ever, one should note that this conclusion, which is drawn from the Navier-Stokes system, is validated by the asymptotic theory ${ }^{26}$ and that the latter theory cannot be applied to the present problem because of the discontinuous temperature distribution. Therefore, the situation in the continuum limit is not obvious, though the flow is likely to vanish intuitively. We will discuss this point later.

Figures 6-8 show the isolines of the density, temperature, and pressure in the lower half of the container in the case of $T_{2} / T_{1}=2$ for four values of the Knudsen number $\mathrm{Kn}=5,0.5,0.05$, and 0.005 . The isolines concentrate on the point of discontinuity of the wall temperature. This means that the point is a singular point at which the values of the

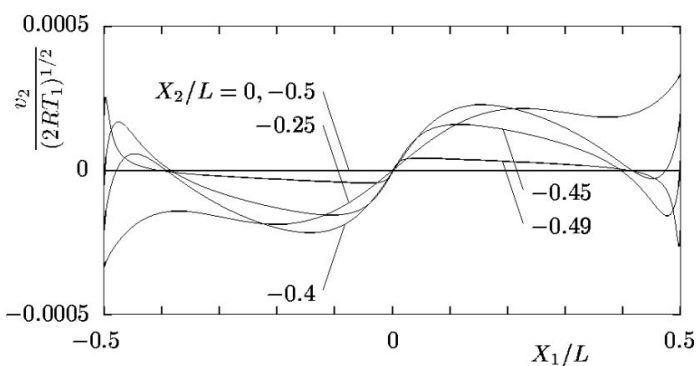

(a) $\mathrm{Kn}=5$

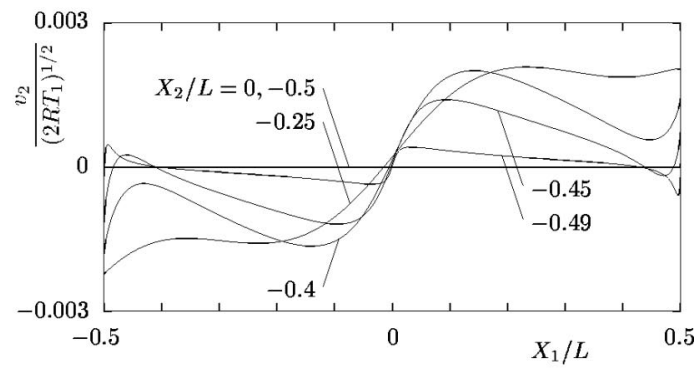

(b) $\mathrm{Kn}=0.5$

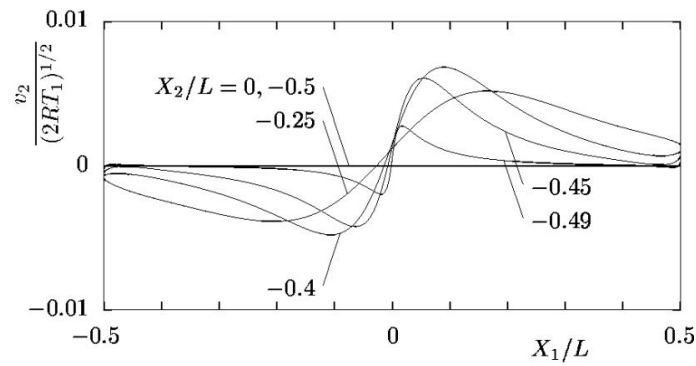

(c) $\mathrm{Kn}=0.05$

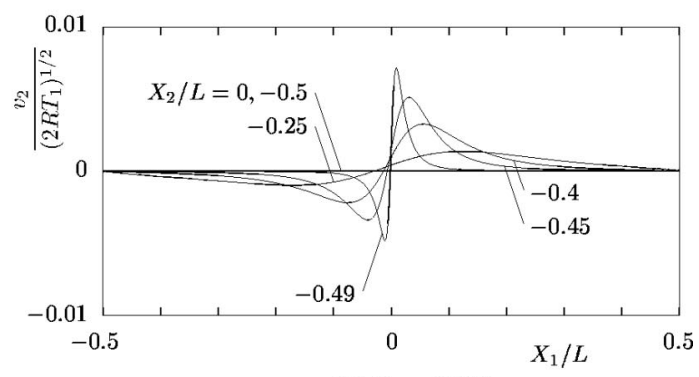

(d) $\mathrm{Kn}=0.005$

FIG. 12. Distributions of the $X_{2}$ component of the flow velocity along the lines $X_{2} / L=$ const for $T_{2} / T_{1}=2$. (a) $\mathrm{Kn}=5$, (b) $\mathrm{Kn}=0.5$, (c) $\mathrm{Kn}=0.05$, (d) $\mathrm{Kn}=0.005$.

macroscopic quantities are not determined uniquely, namely, their limiting values are different depending on the way of approach to the point. For smaller Kn, isodensity and isothermal lines of wider ranges concentrate on the point. The pressure tends to become uniform for small values of Kn [Figs. $8(\mathrm{c})$ and 8(d)]. In Figs. 9-12, we show the distributions of the density, the temperature, and the $X_{1}$ and $X_{2}$ components of the flow velocity along the bottom wall and the lines parallel to it for $\mathrm{Kn}=5,0.5,0.05$, and 0.005 . As is understood from the concentration of the isolines in Figs. 6-8, the distributions on the wall in Figs. 9-12 exhibit a discontinuity at the singular point. The fact that the $X_{1}$ component $v_{1}$ of the flow velocity on the bottom wall $\left(X_{2} / L=-0.5\right)$ does not vanish on the side walls $\left(X_{1} / L= \pm 0.5\right)$ in Figs. 11(a) and 


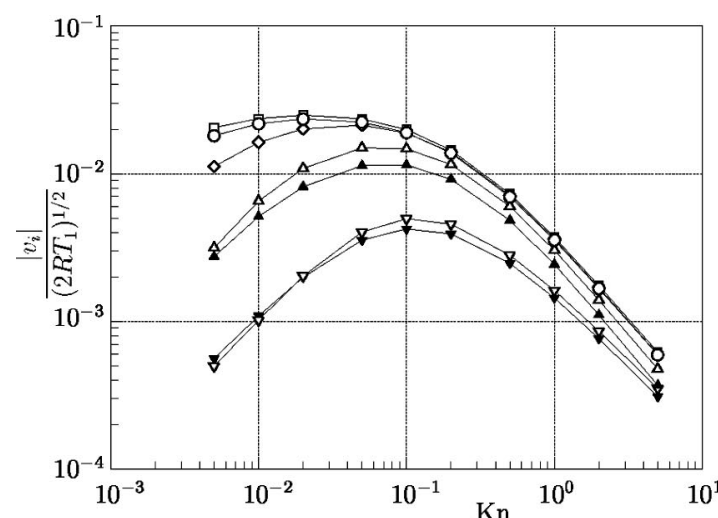

(a)

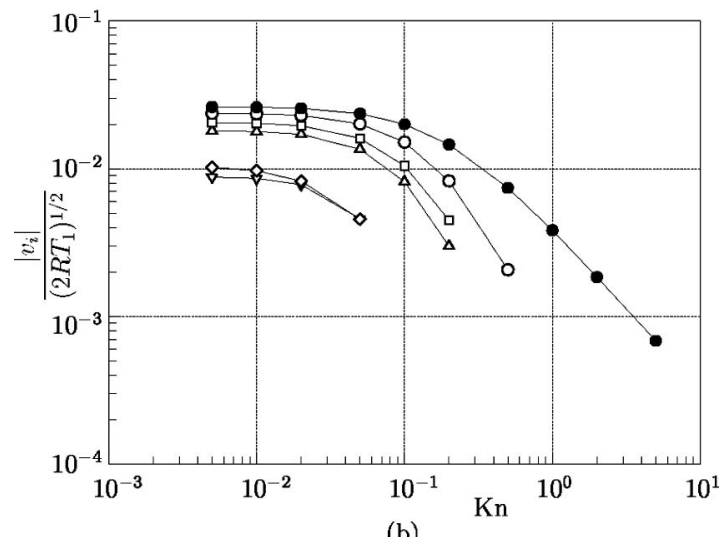

(b)

FIG. 13. Flow speed at various points in the gas vs the Knudsen number for $T_{2} / T_{1}=2$. (a) The flow speed $\left|v_{i}\right|$ at $\left(X_{1} / L, X_{2} / L\right)=(0,-0.495)$ is shown by $\bigcirc,(0.005,-0.495)$ by $\square,(0.02,-0.495)$ by $\diamond,(0.05,-0.495)$ by $\triangle$, $(-0.05,-0.495)$ by $\boldsymbol{\Lambda},(-0.3,-0.4)$ by $\boldsymbol{\nabla}$, and $(0.3,-0.4)$ by $\nabla$. (b) The flow speed $\left|v_{i}\right|$ at $\left(X_{1} / l_{1},\left(X_{2}+0.5 L\right) / l_{1}\right)=(0.5,0.5)$ is shown by $\bigcirc,(1,1)$ by $\square,(5,5)$ by $\diamond,(2,1)$ by $\triangle$, and $(5,1)$ by $\nabla$; the symbol $\bullet$ indicates the maximum speed in the domain. In each figure, the same symbols are joined by straight lines.

11(b) [see also Figs. 3(a) and 3(b)] might seem to be unphysical. However, as is seen from the discussions in Sec. III A, the four corners of the container are also singular points at which the macroscopic quantities are not determined uniquely, i.e., their limiting values there are different depending on the way of approach. In Figs. 9-12 as well as in Figs. 3 and 4, the limiting values along the bottom wall $\left[X_{1} / L= \pm(0.5-0), X_{2} / L=-0.5\right]$ are shown as the values at the corners. Therefore, the nonzero $v_{1}$ appears there. This does not contradict the condition that there is no net mass flow across the wall of the container because the limiting values of the flow velocity at the corners are finite though they are not unique (note that if a point is a source or sink of the mass, the flow speed should be infinite there).

Figure 13(a) illustrates the variation with Kn of the flow speed of the gas at five fixed points on the line $X_{2} / L$ $=-0.495$ near the point of discontinuity, i.e. $\left(X_{1} / L, X_{2} / L\right)=(0,-0.495), \quad(0.005,-0.495), \quad(0.02$, $-0.495),(0.05,-0.495)$, and $(-0.05,-0.495)$, and at two points $\left(X_{1} / L, X_{2} / L\right)=(-0.3,-0.4)$ and $(0.3,-0.4)$, whereas Fig. 13(b) illustrates that at five points the positions of which change depending on Kn but are the same in the

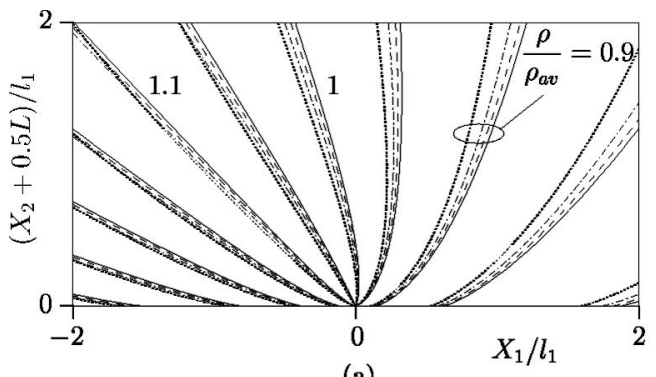

(a)

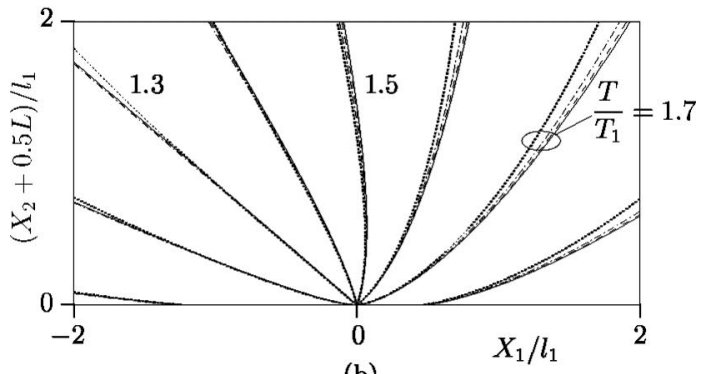

(b)

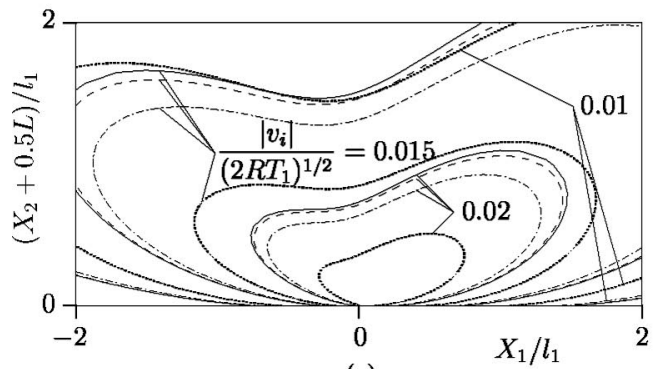

(c)

FIG. 14. Isolines of the density, temperature, and flow speed near the point of discontinuity at small Knudsen numbers for $T_{2} / T_{1}=2$. (a) Density, (b) temperature, (c) flow speed. Here, the dotted line indicates the result for $\mathrm{Kn}=0.05$, the dot-dashed line for $\mathrm{Kn}=0.02$, the dashed line for $\mathrm{Kn}$ $=0.01$, and the solid line for $\mathrm{Kn}=0.005$. The coordinates are normalized by the mean free path $l_{1}$.

scale of the mean free path $l_{1}$ relative to the point of discontinuity, i.e. $\left(X_{1} / l_{1},\left(X_{2}+0.5 L\right) / l_{1}\right)=(0.5,0.5), \quad(1,1)$, $(5,5),(2,1)$, and $(5,1)$. The maximum speed in the flow field is also shown in Fig. 13(b). It is seen from Fig. 13(a) that, as the Knudsen number decreases from 5 to 0.005 , the flow speed at each fixed point first increases, reaches the maximum, and then decreases. The maximum is attained at smaller $\mathrm{Kn}$ for the point closer to the point of discontinuity. On the other hand, in Fig. 13(b), the speed at each point as well as the maximum speed in the flow field tends to approach a finite value as the Knudsen number vanishes. This fact indicates that the flow field near the point of discontinuity for small Kn exhibits a similarity in the sense that the structure of the field expressed in the scale of the mean free path approaches a limiting field as Kn tends to zero. This is also supported by Fig. 14, where the isolines of the density, temperature, and flow speed near the point of discontinuity at $\mathrm{Kn}=0.05,0.02,0.01$, and 0.005 are shown in the coordinates normalized by $l_{1}$. To summarize, the numerical results presented so far, in particular, Figs. 13 and 14, support the following conclusion for the behavior of the flow induced in the gas in the continuum limit. As the limit is approached, 


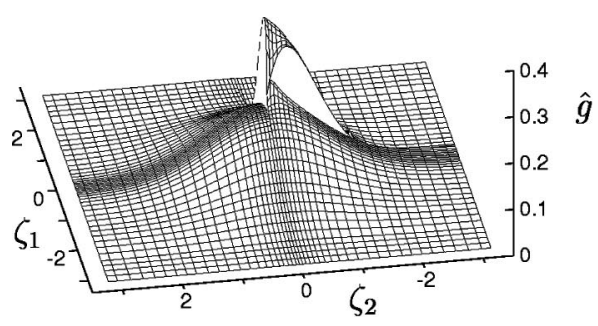

(a) $\left(X_{1} / L, X_{2} / L\right)=(0.101,-0.5)$

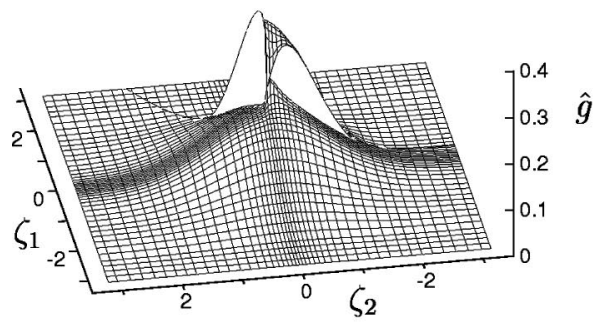

(b) $\left(X_{1} / L, X_{2} / L\right)=(0.101,-0.442)$

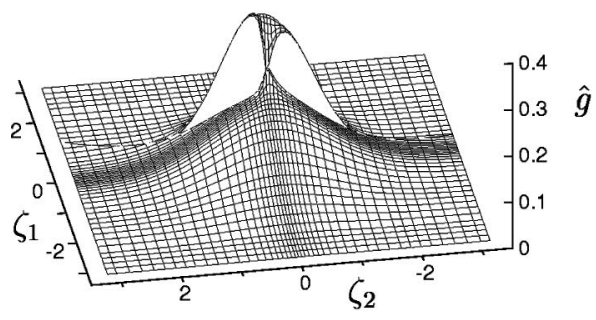

(c) $\left(X_{1} / L, X_{2} / L\right)=(0.101,-0.250)$

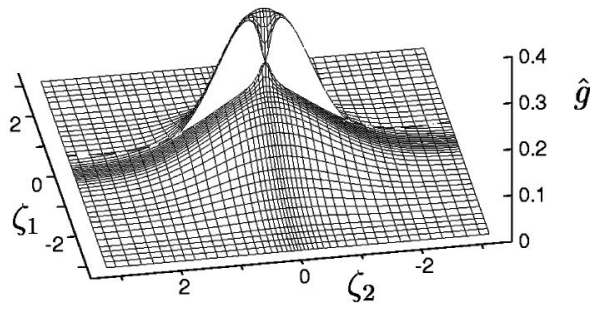

(d) $\left(X_{1} / L, X_{2} / L\right)=(0.101,0)$

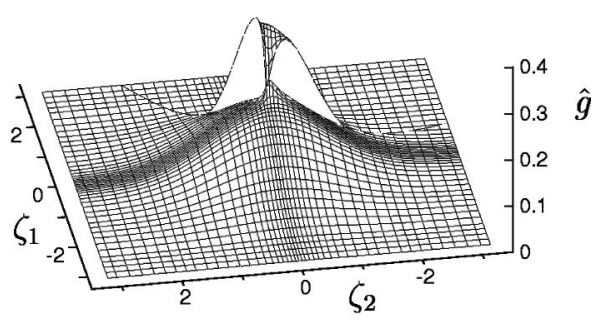

(e) $\left(X_{1} / L, X_{2} / L\right)=(0.25,-0.349)$

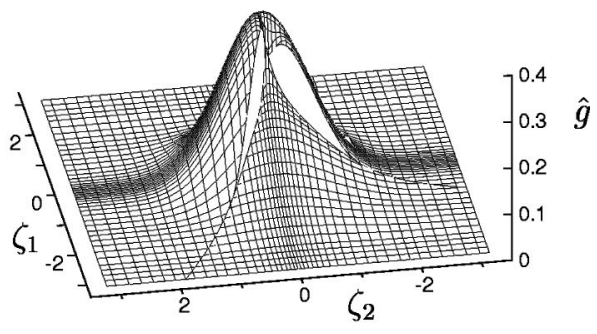

(f) $\left(X_{1} / L, X_{2} / L\right)=(-0.25,-0.349)$
FIG. 15. Dimensionless marginal velocity distribution function $\hat{g}$ [Eq. (28)] at six points in the gas for $\mathrm{Kn}$ $=5$ and $T_{2} / T_{1}=2$. (a) $\left(X_{1} / L, X_{2} / L\right)$ $=(0.101,-0.5), \quad$ (b) $\quad(0.101$, $-0.442), \quad$ (c) $(0.101,-0.250), \quad(\mathrm{d})$ $(0.101,0), \quad$ (e) $(0.25,-0.349), \quad$ (f) $(-0.25,-0.349)$. the region where the flow is appreciable shrinks and converges to the singular point where the wall temperature is discontinuous. That is, as Kn tends to zero, the flow vanishes at all the points except this singular point. In this way, the flow induced in the container vanishes nonuniformly in the continuum limit.

\section{B. Velocity distribution function}

Next, we show the behavior of the velocity distribution function. The dimensionless marginal velocity distribution function $\hat{g}$ introduced in Sec. II D, i.e.,

$$
\begin{aligned}
\hat{g}\left(x_{1}, x_{2}, \zeta_{1}, \zeta_{2}\right) & =\int_{-\infty}^{\infty} \hat{f}\left(x_{1}, x_{2}, \zeta_{i}\right) d \zeta_{3} \\
& =\frac{2 R T_{1}}{\rho_{\mathrm{av}}} \int_{-\infty}^{\infty} f\left(X_{1}, X_{2}, \xi_{i}\right) d \xi_{3},
\end{aligned}
$$

at various points in the gas in the case of $T_{2} / T_{1}=2$ is shown in Figs. $15-18$ as a function of $\zeta_{1}\left[=\xi_{1} /\left(2 R T_{1}\right)^{1 / 2}\right]$ and $\zeta_{2}$ $\left[=\xi_{2} /\left(2 R T_{1}\right)^{1 / 2}\right]$; Figs. $15-18$ correspond to the case of $\mathrm{Kn}=5,0.5,0.05$, and 0.005, respectively. The actual computation has been performed by the use of the lattice lines that are much denser than those drawn on the surface of $\hat{g}$ (see Sec. IV C). The figures (a)-(d) in Figs. 15 and 16 and the figures (a)-(c) in Figs. 17 and 18 are the results at the points almost on the line $X_{1} / L=0.1$, the figure (a) being those on the bottom wall. On the boundary, the velocity distribution function is, in general, discontinuous along the molecular velocities that are tangent to the boundary. As described in Sec. III A, in the present problem, the velocity distribution function in the gas is also discontinuous in the two directions, $\xi_{1} / \xi_{2}=X_{1} /\left(X_{2}+L / 2\right.$ ) (for $\xi_{2}>0$ ) and $\xi_{1} / \xi_{2}=X_{1} /\left(X_{2}-L / 2\right.$ ) (for $\left.\xi_{2}<0\right)$, in the $\xi_{1} \xi_{2}$ plane, originating from the discontinuity of the temperature of the bottom wall and that of the top wall, respectively. In Fig. 15, where the Knudsen number is relatively large $(K n=5)$, the discontinuities in the two directions are large for all the figures. In Fig. 15(a), which shows the velocity distribution on the bottom wall, the discontinuity on the right originates from the temperature discontinuity on the top wall, whereas that on the left is due to the discrepancy between the velocity distributions of impinging and re-emitted molecules. The sharp decay of the discontinuities in the neighborhood of the origin $\left(\zeta_{1}, \zeta_{2}\right)=(0,0)$ is due to the fact that slow molecules have more chances for collision. In Fig. 16, where Kn $=0.5$, the discontinuities are smaller than those at the corresponding spatial positions in Fig. 15 because the effect of molecular collision is larger in the former. In Fig. 17, where 


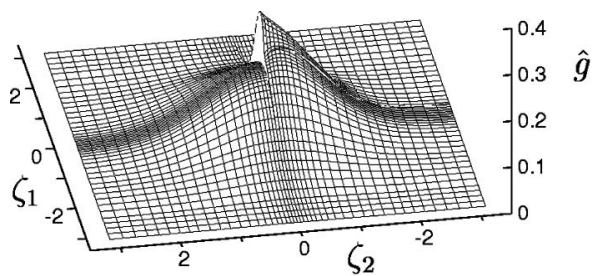

(a) $\left(X_{1} / L, X_{2} / L\right)=(0.101,-0.5)$

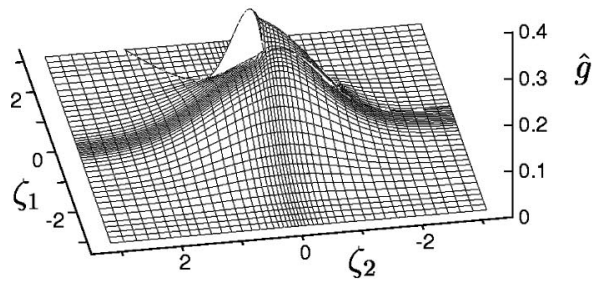

(b) $\left(X_{1} / L, X_{2} / L\right)=(0.101,-0.442)$

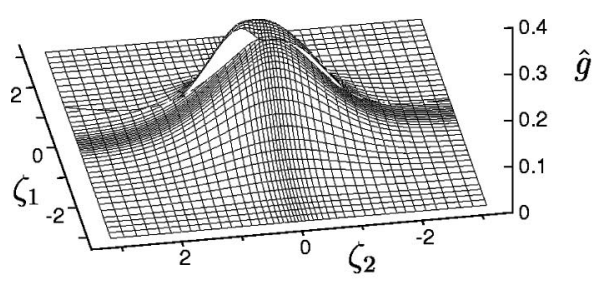

(c) $\left(X_{1} / L, X_{2} / L\right)=(0.101,-0.250)$

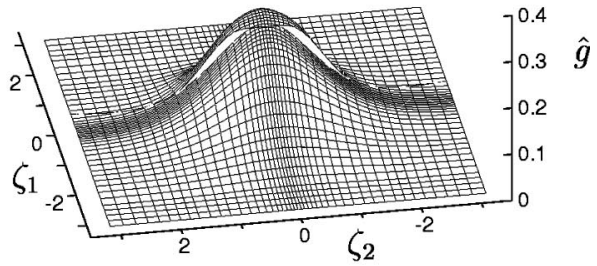

(d) $\left(X_{1} / L, X_{2} / L\right)=(0.101,0)$

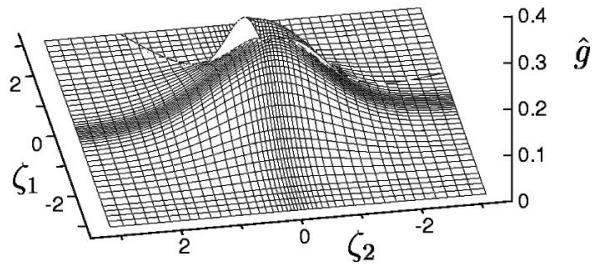

(e) $\left(X_{1} / L, X_{2} / L\right)=(0.25,-0.349)$

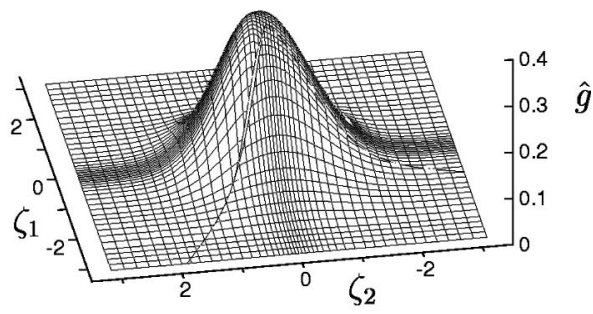

(f) $\left(X_{1} / L, X_{2} / L\right)=(-0.25,-0.349)$
FIG. 16. Dimensionless marginal velocity distribution function $\hat{g}$ [Eq. (28)] at six points in the gas for $\mathrm{Kn}=0.5$ and $T_{2} / T_{1}=2$. (a) $\left(X_{1} / L, X_{2} / L\right)=(0.101,-0.5), \quad($ b) $(0.101,-0.442), \quad$ (c) $\quad(0.101$, $-0.250)$, (d) $(0.101,0)$, (e) $(0.25$, $-0.349)$, (f) $(-0.25,-0.349)$.
$\mathrm{Kn}=0.05$, the discontinuity in the gas originating from the discontinuity of the wall temperature is appreciable only in the close neighborhood of the point of discontinuity [Figs. $17(\mathrm{~d})-17(\mathrm{f})]$. Figure 18 shows $\hat{g}$ for $\mathrm{Kn}=0.005$ at almost the same points as in Fig. 17. The discontinuity is fairly small even in the close neighborhood of the point of discontinuity [Figs. 18(d)-18(f)]. Except in the vicinity of the points of discontinuity on the top and bottom walls, the velocity distribution is close to the local Maxwellian, so that the discontinuity is invisible even on the wall [Fig. 18(a)].

\section{Data on numerical computation}

Finally we give some data on our numerical computation. Here we use the dimensionless space coordinates $\left(x_{1}\right.$, $\left.x_{2}\right)$ and molecular velocity $\left(\zeta_{1}, \zeta_{2}\right)$. The lower half of the container $-1 / 2 \leqslant x_{1} \leqslant 1 / 2,-1 / 2 \leqslant x_{2} \leqslant 0$ is divided into 160 $\times 80(\mathrm{Kn} \geqslant 0.1)$ or $320 \times 160(\mathrm{Kn} \leqslant 0.05)$ nonuniform rectangular regions by lattice lines. For $x_{1}$, the minimum lattice interval is $4.61 \times 10^{-4}(\mathrm{Kn} \geqslant 0.1), 1.93 \times 10^{-4}(\mathrm{Kn}=0.05)$, or $3.42 \times 10^{-3} \mathrm{Kn}(\mathrm{Kn} \leqslant 0.02)$ at $x_{1}=0$ (and also at $x_{1}$ $= \pm 1 / 2$ for $\mathrm{Kn} \geqslant 0.05$; for $\mathrm{Kn} \leqslant 0.02$, the lattice interval at $x_{1}= \pm 1 / 2$ is $\left.2.19 \times 10^{-4}\right)$, and the maximum interval is $1.20 \times 10^{-2} \quad(\mathrm{Kn} \geqslant 0.1), 6.06 \times 10^{-3} \quad(\mathrm{Kn}=0.05)$, or 6.73 $\times 10^{-3}(\mathrm{Kn} \leqslant 0.02)$ at $x_{1}= \pm 1 / 4$. For $x_{2}$, the minimum lattice interval is $3.87 \times 10^{-4}(\mathrm{Kn} \geqslant 0.1), 1.75 \times 10^{-4}(\mathrm{Kn}$
$=0.05)$, or $3.42 \times 10^{-3} \mathrm{Kn}(\mathrm{Kn} \leqslant 0.02)$ at $x_{2}=-1 / 2$, and the maximum interval is $1.21 \times 10^{-2}(\mathrm{Kn} \geqslant 0.1), 6.08 \times 10^{-3}$ $(\mathrm{Kn}=0.05)$, or $5.69 \times 10^{-3} \sim 6.61 \times 10^{-3}(\mathrm{Kn} \leqslant 0.02)$ at $x_{2}$ $=0$. The molecular velocity space $\left(\zeta_{1}, \zeta_{2}\right)$ is restricted to a finite domain $-7.1 \leqslant \zeta_{1} \leqslant 7.1,-7.0 \leqslant \zeta_{2} \leqslant 7.0$ (i.e., $Z_{1}=7.1$ and $\left.Z_{2}=7.0\right)$, which is divided into $320 \times 320(\mathrm{Kn} \geqslant 0.1)$ or $160 \times 160(\mathrm{Kn} \leqslant 0.05)$ nonuniform rectangular regions by lattice lines. For $\zeta_{1}$, the minimum lattice interval is 4.44 $\times 10^{-5}(\mathrm{Kn} \geqslant 0.1)$ or $8.89 \times 10^{-5}(\mathrm{Kn} \leqslant 0.05)$ at $\zeta_{1}=0$, and the maximum interval is $1.76 \times 10^{-1}(\mathrm{Kn} \geqslant 0.1)$ or 3.48 $\times 10^{-1}(\mathrm{Kn} \leqslant 0.05)$ at $\zeta_{1}= \pm 7.1$. For $\zeta_{2}$, the minimum lattice interval is $4.55 \times 10^{-5}(\mathrm{Kn} \geqslant 2), 4.39 \times 10^{-4}(0.1 \leqslant \mathrm{Kn}$ $\leqslant 1)$, or $8.89 \times 10^{-4}(\mathrm{Kn} \leqslant 0.05)$ at $\zeta_{2}=0$, and the maximum interval is $1.30 \times 10^{-1}(\mathrm{Kn} \geqslant 0.1)$ or $2.58 \times 10^{-1}(\mathrm{Kn}$ $\leqslant 0.05)$ at $\zeta_{2}= \pm 7.0$.

The lattice systems are chosen carefully on the basis of various numerical tests. An example of such tests is the observation of the change in macroscopic quantities when a coarser (or finer) lattice system is used. Let $\Gamma_{x}$ and $\Gamma_{\zeta}$ denote, respectively, the standard lattice system in $\left(x_{1}, x_{2}\right)$ and that in $\left(\zeta_{1}, \zeta_{2}\right)$ described in the preceding paragraph $\left(\Gamma_{x}\right.$ and $\Gamma_{\zeta}$ are different depending on $\mathrm{Kn}$ ); let $\Gamma_{x}^{(1 / 4)}$ and $\Gamma_{\zeta}^{(1 / 4)}$ denote, respectively, coarser lattice system with about onefourth lattice points corresponding to $\Gamma_{x}$ and that corresponding to $\Gamma_{\zeta}$ (i.e., $\Gamma_{x}^{(1 / 4)}$ and $\Gamma_{\zeta}^{(1 / 4)}$ are obtained by 


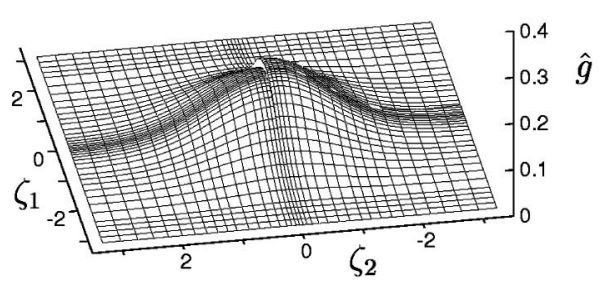

(a) $\left(X_{1} / L, X_{2} / L\right)=(0.101,-0.5)$

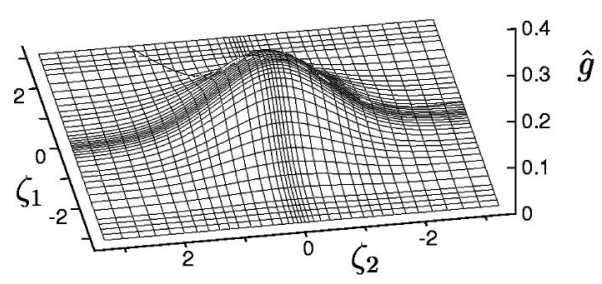

(b) $\left(X_{1} / L, X_{2} / L\right)=(0.101,-0.442)$

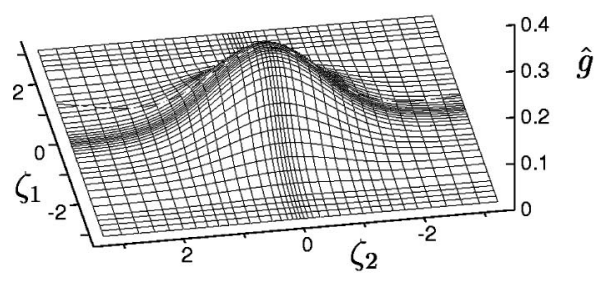

(c) $\left(X_{1} / L, X_{2} / L\right)=(0.101,-0.250)$

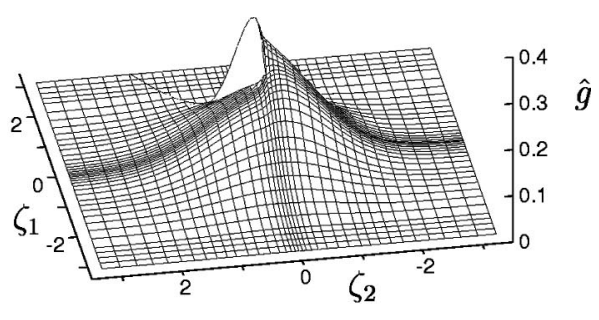

(d) $\left(X_{1} / L, X_{2} / L\right)=(0.005,-0.497)$

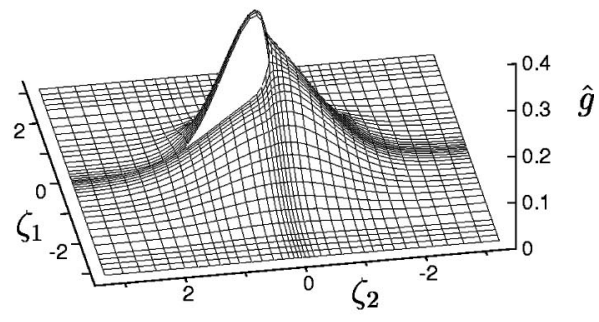

(e) $\left(X_{1} / L, X_{2} / L\right)=(0,-0.497)$

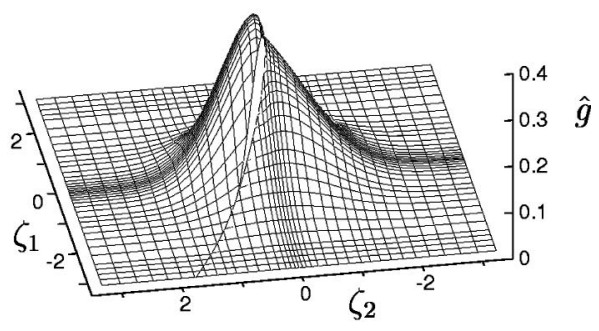

(f) $\left(X_{1} / L, X_{2} / L\right)=(-0.005,-0.497)$
FIG. 17. Dimensionless marginal velocity distribution function $\hat{g}$ [Eq. (28)] at six points in the gas for $\mathrm{Kn}=0.05$ and $T_{2} / T_{1}=2$. (a) $\left(X_{1} / L, X_{2} / L\right)=(0.101,-0.5), \quad($ b) $(0.101,-0.442), \quad$ (c) $\quad(0.101$, $-0.250), \quad$ (d) $(0.005,-0.497), \quad(\mathrm{e})$ $(0,-0.497)$, (f) $(-0.005,-0.497)$. removing every second lattice line in $\Gamma_{x}$ and $\Gamma_{\zeta}$, respectively); and let $\Gamma_{\zeta}^{(4)}$ be a finer lattice system with about four times more lattice points corresponding to $\Gamma_{\zeta}$. For $\mathrm{Kn}$ $=0.5$, the difference between the result based on $\left(\Gamma_{x}, \Gamma_{\zeta}\right)$ and that on $\left(\Gamma_{x}, \Gamma_{\zeta}^{(1 / 4)}\right)$ is less than $1.63 \times 10^{-6}$ in $\hat{\rho}, 4.85$ $\times 10^{-7}$ in $\hat{v}_{1}, 4.51 \times 10^{-7}$ in $\hat{v}_{2}$, and $8.48 \times 10^{-7}$ in $\hat{T}$, and the difference between $\left(\Gamma_{x}, \Gamma_{\zeta}^{(1 / 4)}\right)$ and $\left(\Gamma_{x}^{(1 / 4)}, \Gamma_{\zeta}^{(1 / 4)}\right)$ is less than $3.30 \times 10^{-4}$ in $\hat{\rho}, 1.01 \times 10^{-4}$ in $\hat{v}_{1}, 6.16 \times 10^{-5}$ in $\hat{v}_{2}$, and $8.94 \times 10^{-5}$ in $\hat{T}$. For $\mathrm{Kn}=0.05$, the difference between the result based on $\left(\Gamma_{x}, \Gamma_{\zeta}\right)$ and that on $\left(\Gamma_{x}^{(1 / 4)}, \Gamma_{\zeta}\right)$ is less than $2.31 \times 10^{-4}$ in $\hat{\rho}, 3.02 \times 10^{-4}$ in $\hat{v}_{1}, 4.58 \times 10^{-5}$ in $\hat{v}_{2}$, and $2.66 \times 10^{-4}$ in $\hat{T}$, and the difference between $\left(\Gamma_{x}^{(1 / 4)}, \Gamma_{\zeta}\right)$ and $\left(\Gamma_{x}^{(1 / 4)}, \Gamma_{\zeta}^{(4)}\right)$ is less than $2.99 \times 10^{-6}$ in $\hat{\rho}$, $2.59 \times 10^{-6}$ in $\hat{v}_{1}, 1.65 \times 10^{-6}$ in $\hat{v}_{2}$, and $3.54 \times 10^{-6}$ in $\hat{T}$. For $\mathrm{Kn}=0.005$, the difference between the result based on $\left(\Gamma_{x}, \Gamma_{\zeta}\right)$ and that on $\left(\Gamma_{x}^{(1 / 4)}, \Gamma_{\zeta}^{\prime}\right)$, where $\Gamma_{\zeta}^{\prime}$ is a lattice system with the same number of lattice points as $\Gamma_{\zeta}$ but with slightly different lattice intervals, is less than $5.47 \times 10^{-4}$ in $\hat{\rho}, 5.62 \times 10^{-4}$ in $\hat{v}_{1}, 2.58 \times 10^{-4}$ in $\hat{v}_{2}$, and $7.18 \times 10^{-4}$ in $\hat{T}$, and the difference between $\left(\Gamma_{x}^{(1 / 4)}, \Gamma_{\zeta}^{\prime}\right)$ and $\left(\Gamma_{x}^{(1 / 4)}, \Gamma_{\zeta}^{\prime(4)}\right)$ is less than $6.60 \times 10^{-5}$ in $\hat{\rho}, 7.82 \times 10^{-6}$ in $\hat{v}_{1}, 6.09 \times 10^{-6}$ in $\hat{v}_{2}$, and $1.17 \times 10^{-4}$ in $\hat{T}$.

A convenient measure of the numerical error is provided by the conservation laws. Let us consider a rectangular domain, $-r_{1} \leqslant x_{1} \leqslant r_{1},-0.5 \leqslant x_{2} \leqslant-0.5+r_{2}$, in contact with the bottom wall. Let the bottom side $\left(-r_{1} \leqslant x_{1} \leqslant r_{1}, x_{2}\right.$ $=-0.5)$ be denoted by side I, the top side $\left(-r_{1} \leqslant x_{1} \leqslant r_{1}\right.$, $\left.x_{2}=-0.5+r_{2}\right)$ by side II, the left side $\left(x_{1}=-r_{1}\right.$, $\left.-0.5 \leqslant x_{2} \leqslant-0.5+r_{2}\right)$ by side III, and the right side $\left(x_{1}=r_{1}, \quad-0.5 \leqslant x_{2} \leqslant-0.5+r_{2}\right)$ by side IV. Further, let $\quad \rho_{\mathrm{av}}\left(2 R T_{1}\right)^{1 / 2} L \hat{M}^{(\mathcal{N})}, \quad \rho_{\mathrm{av}} R T_{1} L \hat{P}_{i}^{(\mathcal{N})}, \quad$ and $\quad\left(\rho_{\mathrm{av}} / 2\right)$ $\times\left(2 R T_{1}\right)^{3 / 2} L \hat{E}^{(\mathcal{N})}$ be, respectively, the mass, the $x_{i}$ component of the momentum, and the energy flowing out from the rectangular domain through the side $\mathcal{N}(\mathcal{N}=$ I, II, III, or IV) (thus, $\hat{M}^{(\mathcal{N})}, \hat{P}_{i}^{(\mathcal{N})}$, and $\hat{E}^{(\mathcal{N})}$ are the corresponding dimensionless quantities). The sums of these quantities over the all sides, $\quad \hat{M}_{T}=\sum_{\mathcal{N}=\mathrm{I}}^{\mathrm{IV}} \hat{M}^{(\mathcal{N})}, \quad \hat{P}_{i T}=\sum_{\mathcal{N}=\mathrm{I}}^{\mathrm{IV}} \hat{P}_{i}^{(\mathcal{N})}, \quad$ and $\quad \hat{E}_{T}$ $=\sum_{\mathcal{N}=\mathrm{I}}^{\mathrm{IV}} \hat{E}^{(\mathcal{N})}$, should vanish theoretically because of the conservation of mass, momentum, and energy. However, these do not vanish in the actual computation because of the numerical error. These nonzero values give a convenient measure of accuracy. For example, for $\mathrm{Kn}=0.5, r_{1}=0.204$, and $r_{2}=0.201$, we have $\hat{M}_{T}=3.65 \times 10^{-8} \quad\left(\hat{M}^{(\mathrm{III})}=-5.40\right.$ $\times 10^{-4}$ for reference), $\hat{P}_{1 T}=1.98 \times 10^{-6} \quad\left(\hat{P}_{1}^{(\mathrm{III})}=-2.80\right.$ $\left.\times 10^{-1}\right), \hat{P}_{2 T}=-3.76 \times 10^{-7}\left(\hat{P}_{2}^{(\mathrm{II})}=5.84 \times 10^{-1}\right)$, and $\hat{E}_{T}$ $=-1.91 \times 10^{-6}\left(\hat{E}^{(\mathrm{III})}=5.44 \times 10^{-2}\right) ;$ for $\mathrm{Kn}=0.05, \quad r_{1}$ 


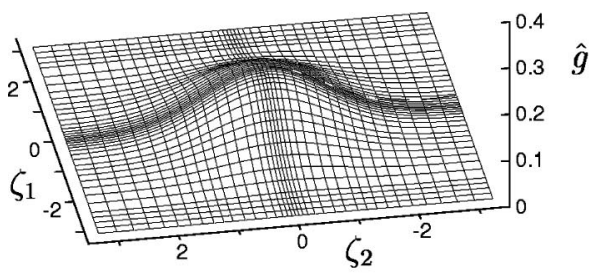

(a) $\left(X_{1} / L, X_{2} / L\right)=(0.100,-0.5)$

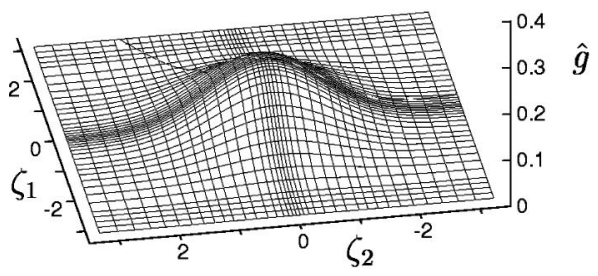

(b) $\left(X_{1} / L, X_{2} / L\right)=(0.100,-0.440)$

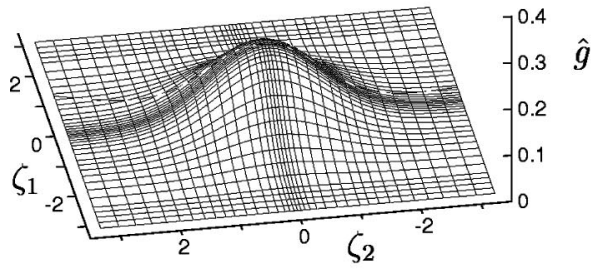

(c) $\left(X_{1} / L, X_{2} / L\right)=(0.100,-0.249)$

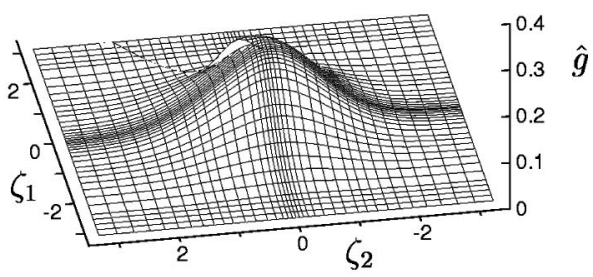

(d) $\left(X_{1} / L, X_{2} / L\right)=(0.005,-0.497)$

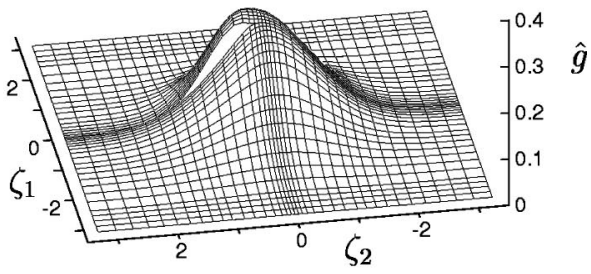

(e) $\left(X_{1} / L, X_{2} / L\right)=(0,-0.497)$

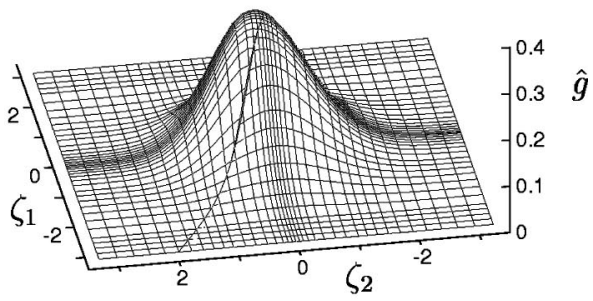

(f) $\left(X_{1} / L, X_{2} / L\right)=(-0.005,-0.497)$
FIG. 18. Dimensionless marginal velocity distribution function $\hat{g}$ [Eq. (28)] at six points in the gas for $\mathrm{Kn}$ $=0.005$ and $T_{2} / T_{1}=2$. (a) $\left(X_{1} / L, X_{2} / L\right)=(0.100,-0.5), \quad($ b) $(0.100,-0.440), \quad$ (c) $\quad(0.100$, $-0.249), \quad(\mathrm{d}) \quad(0.005,-0.497), \quad(\mathrm{e})$ $(0,-0.497)$, (f) $(-0.005,-0.497)$.
$=0.204, \quad$ and $r_{2}=0.201$, we have $\hat{M}_{T}=-2.05 \times 10^{-7}$ $\left(\hat{M}^{(\mathrm{III})}=-1.00 \times 10^{-3}\right), \quad \hat{P}_{1 T}=4.43 \times 10^{-7} \quad\left(\hat{P}_{1}^{(\mathrm{III})}=-2.92\right.$ $\left.\times 10^{-1}\right), \hat{P}_{2 T}=-1.47 \times 10^{-6}\left(\hat{P}_{2}^{(\mathrm{II})}=5.93 \times 10^{-1}\right)$, and $\hat{E}_{T}$ $=-1.27 \times 10^{-6} \quad\left(\hat{E}^{(\mathrm{IV})}=-9.98 \times 10^{-3}\right) ;$ and for $\mathrm{Kn}$ $=0.005, r_{1}=0.200$, and $r_{2}=0.198$, we have $\hat{M}_{T}=-1.00$ $\times 10^{-6}\left(\hat{M}^{(\mathrm{III})}=-1.92 \times 10^{-4}\right), \hat{P}_{1 T}=-3.49 \times 10^{-6}\left(\hat{P}_{1}^{(\mathrm{III})}\right.$ $\left.=-2.90 \times 10^{-1}\right), \hat{P}_{2 T}=-7.42 \times 10^{-7}\left(\hat{P}_{2}^{(\mathrm{II})}=5.86 \times 10^{-1}\right)$, and $\hat{E}_{T}=-1.59 \times 10^{-6}\left(\hat{E}^{(\mathrm{IV})}=-6.20 \times 10^{-4}\right)$.

The present computation was mainly carried out on Cray ORIGIN 2000 computers at the Institute for Chemical Research, Kyoto University.

\section{DISCUSSIONS}

We conclude this paper with some physical discussions about the numerical results. Here we suppose $T_{1}<T_{2}$ as in the numerical analysis.

Let us examine the situation near the point of discontinuity of the wall temperature on the bottom wall for relatively small $\mathrm{Kn}$. The gas in the upper right region with respect to the point of discontinuity is hotter than that in the upper left region, as seen from Figs. 7 and 14(b). Therefore, a flow toward the right is induced in the gas by the same mechanism as the thermal creep flow. ${ }^{1-7}$ Here, we repeat the explanation of the mechanism briefly (see Refs. 22 and 28-
30). Let us suppose that the gas is at rest, and let us consider the molecules incident on a point on the bottom wall near the point of discontinuity. Because the molecules impinging on the point possess the property of the gas in the region about a mean free path apart from the point, the molecules from the upper right region are, on the average, faster than those from the upper left region. Therefore, the incident molecules give the leftward tangential momentum to the wall. Since the molecules leaving the wall, which are isotropically distributed in the case of the diffuse reflection, do not contribute to the transport of the tangential momentum, the leftward tangential momentum is transferred to the wall by the gas molecules. As the reaction, the gas undergoes a force in the rightward direction and moves in the same direction. The speed of the gas motion is determined in such a way that the tangential momentum given to the wall by the gas motion compensates the above-mentioned leftward momentum transferred by the impinging molecules.

Following Refs. 22 and 28-30, we can roughly estimate the speed of the gas motion to be proportional to the temperature difference, say $\Delta T$, between two points that are about a mean free path apart in the horizontal direction and are located about a mean free path above the wall. This estimate applies to the general case with arbitrary temperature distribution. In the case of the usual thermal creep flow caused by an imposed temperature gradient $\partial T_{w} / \partial X_{w}$, 
where $T_{w}$ is the temperature of the boundary and $X_{w}$ is a coordinate along it, the temperature difference $\Delta T$ is given by $l\left(\partial T_{w} / \partial X_{w}\right)$, where $l$ is the reference mean free path. The speed of the gas motion $v$ is thus given as $v$ $\propto l\left(\partial T_{w} / \partial X_{w}\right)$. Therefore, when $l$ goes to zero (the continuum limit), the flow vanishes. In contrast, in the present problem with a discontinuous wall temperature, the temperature field around the point of discontinuity is almost independent of $l$ if it is described in the scale of $l$ [cf. Fig. 14(b)]. Therefore, the temperature difference $\Delta T$ and thus the speed of the gas motion $v$ are almost independent of $l$. This means that the flow does not vanish in the continuum limit. On the other hand, because $\Delta T$ is appreciable only in the small region over a few mean free paths around the point of discontinuity [cf. Fig. 14(b)], the gas flow is driven only locally in this region. Therefore, the region of the flow shrinks to the point of discontinuity as the mean free path approaches zero. In this way, the flow vanishes nonuniformly in the continuum limit.

At small Knudsen numbers, the thermal stress slip flow ${ }^{24,8-11}$ of the order of $\mathrm{Kn}^{2}$ (here we refer to the flow speed normalized by the average molecular speed) and the nonlinear thermal stress flow ${ }^{12,13}$ of the order of Kn, mentioned in Sec. I, should also be induced in the gas. However, the flow caused by the discontinuity of the wall temperature, which is of the order of unity, has a dominant effect even though it is localized.

\section{ACKNOWLEDGMENTS}

The authors thank Toshiyuki Nakanishi for his help in preparing the figures and arranging the numerical data. This work is partially supported by the grants-in-aid (No. 10650171 and No. 10045040) for Scientific Research from the Japan Society for the Promotion of Science.

${ }^{1}$ E. H. Kennard, Kinetic Theory of Gases (McGraw-Hill, New York, 1938). ${ }^{2}$ Y. Sone, "Thermal creep in rarefied gas," J. Phys. Soc. Jpn. 21, 1836 (1966).

${ }^{3}$ S. K. Loyalka, "Slip in the thermal creep flow," Phys. Fluids 14, 21 (1971).

${ }^{4}$ S. K. Loyalka, "Temperature jump and thermal creep slip: Rigid sphere gas," Phys. Fluids A 1, 403 (1989).

${ }^{5}$ T. Ohwada, Y. Sone, and K. Aoki, "Numerical analysis of the shear and thermal creep flows of a rarefied gas over a plane wall on the basis of the linearized Boltzmann equation for hard-sphere molecules," Phys. Fluids A 1, 1588 (1989).

${ }^{6}$ Y. Sone, "A simple demonstration of a rarefied gas flow induced over a plane wall with a temperature gradient," Phys. Fluids A 3, 997 (1991).

${ }^{7}$ Y. Sone, K. Sawada, and H. Hirano, "A simple experiment on the strength of thermal creep flow of a rarefied gas over a flat wall," Eur. J. Mech. B/Fluids 13, 299 (1994).

${ }^{8}$ Y. Sone, "Flow induced by thermal stress in rarefied gas," Phys. Fluids 15, 1418 (1972).

${ }^{9}$ Y. Sone, "Rarefied gas flow induced between non-parallel plane walls with different temperatures," in Rarefied Gas Dynamics, edited by M. Becker and M. Fiebig (DFVLR, Porz-Wahn, Germany, 1974), D.23-1.

${ }^{10}$ Y. Sone and S. Tanaka, "Thermal stress slip flow induced in rarefied gas between noncoaxial circular cylinders," in Theoretical and Applied Mechanics, edited by F. P. J. Rimrott and B. Tabarrok (North-Holland, Amsterdam, 1980), p. 405

${ }^{11}$ T. Ohwada and Y. Sone, "Analysis of thermal stress slip flow and negative thermophoresis using the Boltzmann equation for hard-sphere molecules," Eur. J. Mech. B/Fluids 11, 389 (1992).

${ }^{12}$ V. S. Galkin, M. N. Kogan, and O. G. Fridlander, "Free convection in a gas in the absence of external forces," Fluid Dyn. 6, 448 (1971).

${ }^{13}$ M. N. Kogan, V. S. Galkin, and O. G. Fridlander, "Stresses produced in gasses by temperature and concentration inhomogeneities. New types of free convection," Sov. Phys. Usp. 19, 420 (1976).

${ }^{14} \mathrm{~K}$. Aoki, Y. Sone, and T. Yano, "Numerical analysis of a flow induced in a rarefied gas between noncoaxial circular cylinders with different temperatures for the entire range of the Knudsen number," Phys. Fluids A 1, 409 (1989).

${ }^{15}$ T. Ohwada, Y. Sone, and K. Aoki, "Numerical analysis of the Poiseuille and thermal transpiration flows between two parallel plates on the basis of the Boltzmann equation for hard-sphere molecules," Phys. Fluids A 1, 2042 (1989).

${ }^{16} \mathrm{~S}$. Takata and Y. Sone, "Flow induced around a sphere with a nonuniform surface temperature in a rarefied gas, with application to the drag and thermal force problems of a spherical particle with an arbitrary thermal conductivity," Eur. J. Mech. B/Fluids 14, 487 (1995).

${ }^{17} \mathrm{~J}$. Ibsen, R. Soto, and P. Cordero, "Free thermal convection driven by nonlocal effects," Phys. Rev. E 52, 4533 (1995).

${ }^{18}$ Y. Sone, Y. Waniguchi, and K. Aoki, "One-way flow of a rarefied gas induced in a channel with a periodic temperature distribution," Phys. Fluids 8, 2227 (1996).

${ }^{19} \mathrm{~S}$. Takata and N. Ohtsubo, "Natural convection and thermal creep flow induced along a vertical plate with a temperature gradient," in Rarefied Gas Dynamics, edited by C. Shen (Peking University Press, Beijing, 1997), p. 107.

${ }^{20}$ D. H. Papadopoulos and D. E. Rosner, "Internal flows driven by kinetic boundary layers," in Rarefied Gas Dynamics, edited by C. Shen (Peking University Press, Beijing, 1997), p. 142.

${ }^{21} \mathrm{~K}$. Aoki, Y. Sone, and Y. Waniguchi, "A rarefied gas flow induced by a temperature field: Numerical analysis of the flow between coaxial elliptic cylinders with different uniform temperatures," Comput. Math. Appl. 35, 15 (1998).

${ }^{22}$ Y. Sone, "Flows induced by temperature fields in a rarefied gas and their ghost effect on the behavior of a gas in the continuum limit," in Annual Review of Fluid Mechanics (Annual Reviews, Palo Alto, 2000), Vol. 32, p. 779.

${ }^{23}$ Y. Sone, "Asymptotic theory of flow of rarefied gas over a smooth boundary I," in Rarefied Gas Dynamics, edited by L. Trilling and H. Y. Wachman (Academic, New York, 1969), p. 243.

${ }^{24}$ Y. Sone, "Asymptotic theory of flow of rarefied gas over a smooth boundary II," in Rarefied Gas Dynamics, edited by D. Dini (Editrice Tecnico Scientifica, Pisa, Italy, 1971), Vol. II, p. 737.

${ }^{25}$ Y. Sone, "Asymptotic theory of a steady flow of a rarefied gas past bodies for small Knudsen numbers," in Advances in Kinetic Theory and Continuum Mechanics, edited by R. Gatignol and Soubbaramayer (Springer, Berlin, 1991), p. 19.

${ }^{26}$ Y. Sone, K. Aoki, S. Takata, H. Sugimoto, and A. V. Bobylev, "Inappropriateness of the heat-conduction equation for description of a temperature field of a stationary gas in the continuum limit: Examination by asymptotic analysis and numerical computation of the Boltzmann equation," Phys. Fluids 8, 628 (1996); 8, 841(E) (1996).

${ }^{27}$ Y. Sone, C. Bardos, F. Golse, and H. Sugimoto, "Asymptotic theory of the Boltzmann system for a steady flow of a slightly rarefied gas with a finite Mach number: General theory," Eur. J. Mech. B/Fluids 19, 325 (2000).

${ }^{28}$ Y. Sone and K. Aoki, Molecular Gas Dynamics (Asakura, Tokyo, 1994) (in Japanese).

${ }^{29}$ Y. Sone, "Theoretical and numerical analyses of the Boltzmann equation-Theory and analysis of rarefied gas flows-," Lecture Notes (Department of Aeronautics and Astronautics, Graduate School of Engineering, Kyoto University, 1998), Part I (http://www.users.kudpc.kyotou.ac.jp/ a50077).

${ }^{30}$ Y. Sone, Kinetic Theory and Fluid Dynamics, Modeling and Simulation in Science, Engineering and Technology (Birkhäuser, Basel, in press).

${ }^{31} \mathrm{~K}$. Aoki, Y. Sone, and N. Masukawa, "A rarefied gas flow induced by a temperature field," in Rarefied Gas Dynamics, edited by J. Harvey and G. Lord (Oxford University Press, Oxford, 1995), p. 35.

${ }^{32}$ Y. Sone and M. Yoshimoto, "Demonstration of a rarefied gas flow induced near the edge of a uniformly heated plate," Phys. Fluids 9, 3530 (1997).

${ }^{33}$ G. A. Bird, Molecular Gas Dynamics (Oxford University Press, Oxford, 1976).

${ }^{34}$ G. A. Bird, Molecular Gas Dynamics and the Direct Simulation of Gas Flows (Oxford University Press, Oxford, 1994).

${ }^{35}$ Some of the results were presented at the Fourth International Congress on Industrial and Applied Mathematics, Edinburgh, Scotland, July 5-9, 1999 
(K. Aoki, Y. Sone, K. Ueda, and S. Takata, "Numerical analysis of a rarefied gas flow induced near the edges of a uniformly cooled or heated plate," Book of Abstracts, p. 155); see also K. Ueda, Master's thesis, Department of Aeronautics and Astronautics, Graduate School of Engineering, Kyoto University, 1999 (in Japanese).

${ }^{36}$ P. L. Bhatnagar, E. P. Gross, and M. Krook, "A model for collision processes in gases. I. Small amplitude processes in charged and neutral onecomponent systems," Phys. Rev. 94, 511 (1954).

${ }^{37} \mathrm{P}$. Welander, "On the temperature jump in a rarefied gas," Ark. Fys. 7, 507 (1954).

${ }^{38}$ M. N. Kogan, "On the equations of motion of a rarefied gas," Appl. Math. Mech. 22, 597 (1958).

${ }^{39}$ Y. Sone, "Continuum gas dynamics in the light of kinetic theory and new features of rarefied gas flows," in Rarefied Gas Dynamics, edited by C. Shen (Peking University Press, Beijing, 1997), p. 3

${ }^{40}$ Y. Sone, S. Takata, and H. Sugimoto, "The behavior of a gas in the continuum limit in the light of kinetic theory: The case of cylindrical Couette flows with evaporation and condensation," Phys. Fluids 8, 3403 (1996); 10, 1239(E) (1998).

${ }^{41} \mathrm{~S}$. Takata and K. Aoki, "Two-surface problems of a multicomponent mixture of vapors and noncondensable gases in the continuum limit in the light of kinetic theory," Phys. Fluids 11, 2743 (1999).

${ }^{42}$ S. Takata, K. Aoki, and T. Muraki, "Behavior of a vapor-gas mixture between two parallel plane condensed phases in the continuum limit," in Rarefied Gas Dynamics, edited by R. Brun, R. Campargue, R. Gatignol, and J.-C. Lengrand (Cépaduès-Éditions, Toulouse, 1999), Vol. 1, p. 479.

${ }^{43} \mathrm{~S}$. Takata and K. Aoki, "The ghost effect in the continuum limit for a vapor-gas mixture around condensed phases: Asymptotic analysis of the Boltzmann equation," Transp. Theory Stat. Phys. 30, 205 (2001).

${ }^{44}$ F. Bouchut, F. Golse, and M. Pulvirenti, Kinetic Equations and Asymptotic Theory, edited by B. Perthame and L. Desvillettes (Gauthier-Villars, Paris, 2000), Chap. 2.

${ }^{45}$ C. Cercignani, Rarefied Gas Dynamics, From Basic Concepts to Actual Calculations (Cambridge University Press, Cambridge, 2000).

${ }^{46} \mathrm{C}$. K. Chu, "Kinetic-theoretic description of the formation of a shock wave," Phys. Fluids 8, 12 (1965).

${ }^{47}$ Y. Sone and S. Takata, "Discontinuity of the velocity distribution function in a rarefied gas around a convex body and the $\mathrm{S}$ layer at the bottom of the Knudsen layer,” Transp. Theory Stat. Phys. 21, 501 (1992).

${ }^{48}$ Y. Sone, "New kind of boundary layer over a convex solid boundary in a rarefied gas," Phys. Fluids 16, 1422 (1973).
${ }^{49} \mathrm{C}$. Cercignani, "Propagation phenomena in classical and relativistic rarefied gases," Transp. Theory Stat. Phys. 29, 607 (2000).

${ }^{50} \mathrm{~K}$. Aoki, K. Kanba, and S. Takata, "Numerical analysis of a supersonic rarefied gas flow past a flat plate," Phys. Fluids 9, 1144 (1997).

${ }^{51}$ L. Boudin and L. Desvillettes, "On the singularities of the global small solutions of the full Boltzmann equation," Monatsh. Math. 131, 91 (2000).

${ }^{52} \mathrm{~K}$. Aoki, C. Bardos, C. Dogbe, and F. Golse, "A note on the propagation of boundary induced discontinuities in kinetic theory," Math. Models Meth. Appl. Sci. (to be published).

${ }^{53}$ It is possible that the gain term of the collision integral of the Boltzmann equation regularizes the singularity in the velocity distribution function less quickly than that of the BGK model does. Therefore, in the case of the Boltzmann equation, there might exist a term with intermediate regularity between the discontinuous term propagated from the boundary and the continuous term. Such a term, however, would be impossible to be discriminated from others numerically. We need a further mathematical study to clarify this point.

${ }^{54}$ Y. Sone and H. Sugimoto, "Strong evaporation from a plane condensed phase," in Adiabatic Waves in Liquid-Vapor Systems, edited by G. E. A. Meier and P. A. Thompson (Springer, Berlin, 1990), p. 293.

${ }^{55}$ K. Aoki, Y. Sone, K. Nishino, and H. Sugimoto, "Numerical analysis of unsteady motion of a rarefied gas caused by sudden changes of wall temperature with special interest in the propagation of a discontinuity in the velocity distribution function," in Rarefied Gas Dynamics, edited by A. E. Beylich (VCH, Weinheim, 1991), p. 222.

${ }^{56} \mathrm{H}$. Sugimoto and Y. Sone, "Numerical analysis of steady flows of a gas evaporating from its cylindrical condensed phase on the basis of kinetic theory," Phys. Fluids A 4, 419 (1992).

${ }^{57}$ S. Takata, Y. Sone, and K. Aoki, "Numerical analysis of a uniform flow of a rarefied gas past a sphere on the basis of the Boltzmann equation for hard-sphere molecules," Phys. Fluids A 5, 716 (1993).

${ }^{58}$ Y. Sone and H. Sugimoto, "Kinetic theory analysis of steady evaporating flows from a spherical condensed phase into a vacuum," Phys. Fluids A 5, 1491 (1993)

${ }^{59}$ Y. Sone, "Highly rarefied gas around a group of bodies with various temperature distributions. I. Small temperature variation," J. Mec. Theor. Appl. 3, 315 (1984)

${ }^{60} \mathrm{Y}$. Sone, "Highly rarefied gas around a group of bodies with various temperature distributions. II. Arbitrary temperature variation," J. Mec. Theor. Appl. 4, 1 (1985). 